\title{
Managing Psoriatic Arthritis With Inflammatory Bowel Disease and/or Uveitis
}

\author{
Alfred Yu Ting Chia ${ }^{1,2}$, Gladys Wei Xin Ang ${ }^{2}$, Anita Sook Yee Chan ${ }^{1,3}$, Webber Chan ${ }^{4}$, \\ Timothy Kit Yeong Chong ${ }^{5}$ and Ying Ying Leung ${ }^{1,5 *}$
}

${ }^{1}$ Duke-NUS Medical School, Singapore, Singapore, ${ }^{2}$ Translational Immunology Institute, SingHealth Duke-NUS Academic Medical Centre, Duke-NUS Medical School, Singapore, Singapore, ${ }^{3}$ Singapore National Eye Center and Singapore Eye Research Center, Singapore, Singapore, ${ }^{4}$ Department of Gastroenterology and Hepatology, Singapore General Hospital, Singapore, Singapore, ${ }^{5}$ Department of Rheumatology and Immunology, Singapore General Hospital, Singapore, Singapore

Psoriatic arthritis (PSA) is a chronic inflammatory disease that presents with psoriasis (PsO), peripheral and axial arthropathy. The heterogeneity of disease presentation leads to the term "psoriatic disease (PsD)" which is thought to better encompass the range of clinical manifestations. PSA is associated with several comorbidities such as cardiovascular diseases, metabolic syndrome and other extra-articular manifestations including uveitis, and inflammatory bowel disease (IBD). While novel therapeutics are being developed following advances in our understanding of the pathogenesis of the disease, the diverse combinations of PsA with its various comorbidities still pose a clinical challenge in managing patients with PsA. This article reviews our current understanding of the pathogenesis of PsA and how various pathways in the pathogenesis lead to the two comorbid extra-articular manifestations - uveitis and IBD. We also review current evidence of treatment strategies in managing patients with PsA with comorbidities of uveitis and/or IBD.

\section{Keywords: psoriatic arthritis, uveitis, inflammatory bowel disease, co-morbidity, biologic therapy}

\section{INTRODUCTION}

Psoriatic arthritis (PsA) is an inflammatory arthritis associated with psoriasis (PsO) (1). It belongs to the family of spondyloarthritis (SpA) and the musculoskeletal manifestations include peripheral arthritis, dactylitis, enthesitis, and axial arthropathy. The impact of PsA extends beyond skin and joints to disability, fatigue, anxiety, depression, and poor quality of life $(2,3)$. PsA is associated with comorbidities such as obesity, metabolic syndrome, insulin resistance, and cardiovascular disease (4). The extra-articular manifestations of PsA include inflammatory bowel disease (IBD) and uveitis (5). In recent years, there are advancements in therapeutic options to treat musculoskeletal manifestations of PsA (6), but research to understand the pathogenesis of extra-articular manifestations and their treatment options is still in infancy. The purpose of this review is to summarize the current understanding of pathogenesis of PsA and the extra-articular manifestations and their treatment options.

\section{EXTRA-ARTICULAR MANIFESTATIONS}

IBD

Crohn's disease (CD) and ulcerative colitis (UC) are the two main forms of IBD. CD is characterized by chronic, patchy granulomatous inflammation with skip lesions, affecting any part of the 
gastrointestinal tract, especially the terminal ileum and colon. The inflammation is transmural which can lead to fibrosis, stricture, and fistula. In contrast, $\mathrm{UC}$ is characterized by continuous mucosal inflammation extending from the rectum proximally toward the colon. Differentiating these two conditions is important as each has diverse prognoses and differential responses to treatment (7). The clinical presentations of IBD include recurrent abdominal pain, bloody diarrhea, and mucus in the stool. Patients with $\mathrm{CD}$ can present with intestinal obstruction, recurrent fistulas, and other perianal findings. Systemic symptoms include fatigue, weight loss, fever, and symptoms of anemia. The standardized mortality ratio for CD ranges from 1.2 to 1.9 times the general population (8). The prognosis of IBD has improved in recent decades due to therapeutic advances.

Amongst patients with IBD, extraintestinal manifestations are common, including musculoskeletal (axial and peripheral arthropathy and arthritis), ocular (uveitis, scleritis and episcleritis), and skin. Inflammatory arthropathies are reported up to $40 \%$ of patients with IBD (9). While asymptomatic sacroiliitis may be seen in up to three-quarters of IBD patients, the reported prevalence of seronegative SpA ranges from 18$45 \%$, and ankylosing spondylitis (AS) 3-9.9\% (10, 11). Peripheral arthritis is reported in $7-16 \%$ of IBD patients. Peripheral arthritis is mainly asymmetrical and oligoarticular, usually acute and occurs during IBD exacerbations, and self-limiting. However, it may also persist for months or years. Its onset usually coincides with or after IBD but may precede IBD. Enthesitis and dactylitis were reported in $2-4 \%$ of patients (12).

Amongst patients with SpA, IBD is common (13). Patients with $\mathrm{PsO}$, PsA and AS have a 1-4 fold increased risk of IBD compared to the general population (14-18). Among patients with SpA, 30-42\% have endoscopic (macroscopic) gastrointestinal inflammation (19-22) while 46-58 \% have histologic (microscopic) inflammation $(20,21,23)$. The presence of macroscopic or microscopic inflammatory lesions poorly correlates with symptoms (19). In patients with axial SpA, the severity of microscopic inflammation was significantly associated with severity of bone marrow edema on magnetic resonance imaging, indicating a link between mucosal inflammation and progressive disease (24). These subclinical gastrointestinal inflammatory lesions may predispose SpA patients to develop IBD, with a lifetime IBD risk of between 4-8\% (25-28). Among patients with $\mathrm{PsO}$ and PsA, IBD is more common in patients with more severe PsA (29). IBD is also more common in patients with axial-PsA than in those with peripheral-only PsA (30).

\section{Uveitis}

Uveitis is the inflammation of the uveal tract of the eye which comprises of the iris, ciliary body, and choroid. Adjacent structures including retina, optic nerve, vitreous, and sclera may also be affected. Clinically, uveitis is categorized anatomically - anterior, intermediate, posterior, or panuveitis (31). There is an increased association of ocular manifestations amongst patients with $\operatorname{PsD}(32,33)$. Other presentations like vitritis, retinal vasculitis, and cystoid macular edema involving the posterior chamber are sight-threatening $(34,35)$. The prevalence of uveitis increases with disease duration, lifelong prevalence is over $40 \%$. Among patients with $\mathrm{SpA}$, acute anterior uveitis (AAU) is most common (26) and its prevalence varied with the type of SpA: $33 \%$ in AS; $37 \%$ in IBD-associated arthritis; $26 \%$ in reactive arthritis; $25 \%$ in PsA; and $13 \%$ in undifferentiated SpA $(36,37)$. In both Asian and Western populations, uveitis is common in patients with severe PsO and those with PsA $(38,39)$. Uveitis in SpA usually presents with a 'unilateral alternating' pattern, sudden in onset, confined to the anterior chamber, and completely resolves between episodes (40). In contrast, uveitis in PsA is insidious in onset, bilateral with a chronic relapsing course. PsA patients with both uveitis and axial arthropathy tend to be male and HLA-B*27 positive $(41,42)$. HLA-DR* 13 positivity is also associated with uveitis in patients with PsA (43). Uveitis may also precede the development of PsA in patients with PsO (44).

\section{PATHOGENESIS}

\section{Pathogenesis of PsA}

A combination of genetic and environmental factors contributes to pathogenesis of PsA (Figure 1). Genetic component in PsA is strong (45). HLA class I alleles such as HLA-B*27:05:02 haplotype is widely reported to be positively associated with enthesitis, dactylitis, and sacroiliitis while the HLA$\mathrm{B}^{*}$ 08:01:01-HLA-C*07:01:01 haplotype is positively associated with joint fusion, deformity and asymmetrical sacroiliitis. In contrast, the $B^{*} 44: 03: 01-C^{*} 16: 01: 01$ haplotype may be protective against enthesitis (46). Additional HLA haplotypes associated with susceptibility to PsA were HLA-B*38, and HLA-B*39 (47-51). Non-HLA PsA susceptibility loci related to inflammatory pathways have been implicated. IL-23 receptor (IL23R) polymorphisms are associated with risk of PsA (52). Tumor necrosis factor receptor-associated factor 3 -interacting protein 2 (TRAF3IP2), encoding nuclear factor- $\kappa \mathrm{B}(\mathrm{NF \kappa} \mathrm{B})$ activator protein 1 (Act1) which is an adaptor protein for interleukin-17 (IL-17) receptor (53-55), IL-23A, IL-12B, and TYK2 (Tyrosine Kinase 2) are other examples, highlighting the importance of IL-23/IL-17 axis in the pathogenesis of PsA (56).

In a genetically predisposed individual, environmental factors including mechanical stress may trigger enthesitis - a hallmark clinical presentation of SpA including PsA $(57,58)$. Mechanical stress and trauma release damage-associated molecular patterns (DAMPs), triggering the production of prostaglandin E2 (PGE2) (59) by resident mesenchymal cells, which recruit innate immune cells to perpetuate inflammation. PGE2 also induces $\mathrm{T}$ cell secretion of IL-17, a key driver in PsA pathogenesis $(58,60)$. Innate immune cells such as dendritic cells (DCs), monocytes/macrophages, neutrophils, and innate lymphoid cells (ILCs) corroborate with adaptive immune cells to perpetuate inflammation in PsA (61). Additionally, plasmacytoid dendritic cells ( $\mathrm{pDCs}$ ) infiltrate the synovium to act as antigen presenting cells (APCs), triggering downstream expression of TNF- $\alpha$, IFN$\gamma$, and IL-2 from CD68+ macrophage-like-synoviocytes that mediate synovial inflammation and bone erosions $(62,63)$. TNF $\alpha$ synergizes with DCs to activate and polarize Th17 cells (64). In addition to Th17 cells, type 3 innate lymphoid cells (ILCs) $(65,66)$, mucosal-associated variant T (MAIT) cells $(67,68)$, and 


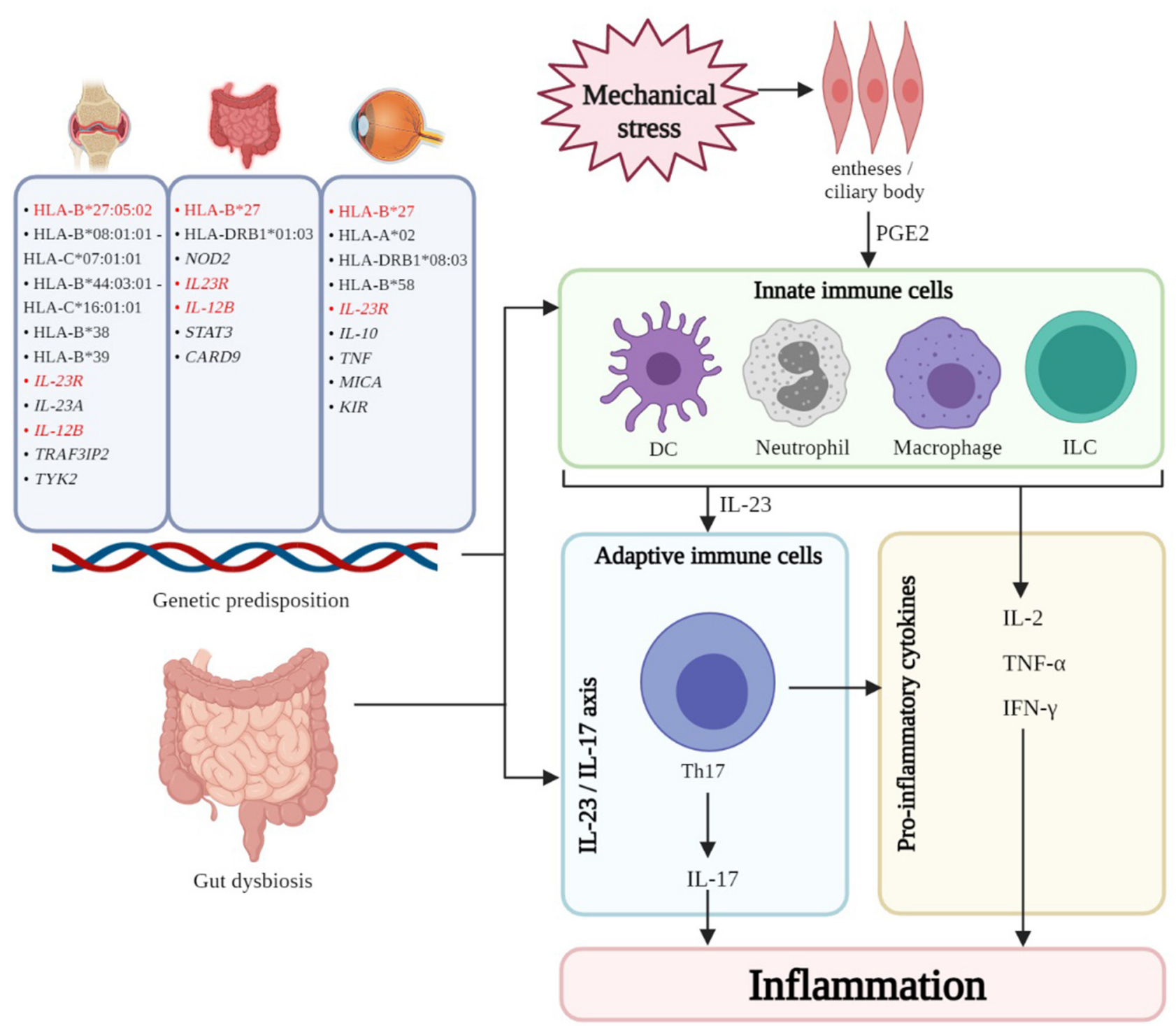

FIGURE 1 | The interplay of genetic, immune, and other factors results in inflammation of the various domains - skin, joints, gut, and eye - in PsD. Common genetic associations (highlighted in red) can be found amongst the three manifestations. Gut dysbiosis is thought to contribute to the pathogenesis for the three manifestations by leakage of bacterial antigen into systemic circulation thereby resulting in inflammation and/or trafficking of immune subsets to and from the Gl mucosa and other sites. In PSA enthesitis and uveitis, mechanical stress triggers the release of PGE2, resulting in the recruitment and activation of innate immune cells (DC, neutrophils, macrophages, ILC like type 3 ILC, MAIT cells, $\gamma \delta$ T cells) which perpetuate inflammation. Furthermore, these innate cells secrete cytokines, notably IL-23, which polarize and maintain Th17 cells which are central to the IL-23/LL-17 axis which is believed to be important in the pathogenesis of PsD. HLA, human leukocyte antigen; IL-23R, interleukin-23 receptor; IL-23A, interleukin-23A; IL-12B, interleukin-12B; TRAF3IP2, tumor necrosis factor receptor-associated factor 3-interacting protein 2; TYK2, tyrosine kinase 2; NOD2, nucleotide-binding oligomerization domain-containing protein 2; STAT3, signal transducer and activator of transcription 3; CARD9, caspase recruitment domain family Member 9; TNF, tumor necrosis factor; MICA, major histocompatibility complex class I chain-related gene A; KIR, killer immunoglobulin receptor; DC, dendritic cell; ILC, innate lymphoid cell; MAIT, mucosal-associated invariant T cell; Th17, T helper 17 cell; IL-17, interleukin-17; IL-2, interleukin-2; TNF- $\alpha$, tumor necrosis factor alpha; IFN- $\gamma$, interferon gamma. Created with BioRender.com.

$\gamma \delta$ T cells (69) are recruited to the synovium and produce IL-17A upon stimulation (70). In short, the IL-23/IL-17 axis is the central driver of inflammation in PsA (71-75).

\section{Pathogenesis of IBD}

Genetic predisposition increases the risk of developing IBD amongst patients with PsA and SpA. HLA-B27 is the major HLA associated with IBD risk. $25-78 \%$ of patients with AS and IBD are HLA-B27 positive $(9,76,77)$. HLA-DRB1*01:03 is also common between AS and IBD (76, 78-80). Non-HLA polymorphisms such as nucleotide-binding oligomerization domain-containing protein 2 (NOD2) polymorphisms increase the risk of CD about 4-40 times and is associated with sacroiliitis amongst patients with IBD. NOD2, an intracellular receptor expressed by immune 
and intestinal cells, is involved in the activation of NFKB and inducing pro-inflammatory genes in innate immune cells (81$85) . I L-23 R$ polymorphisms modify susceptibility to IBD, where a loss-of-function mutation may have protective effect against IBD (86). Polymorphisms in genes related to the IL-23/IL-17 axis such as $I L-12 B$, signal transducer and activator of transcription 3 (STAT3), and caspase recruitment domain family member 9 (CARD9) are associated with CD (87). Once again, this highlights the IL-23/IL-17 axis as a major pathogenetic pathway for IBD manifestations in patients with PsA.

The microbiome plays an important role in gastrointestinal health, and dysbiosis of the microbiota is observed in patients with IBD. Microbiota in IBD patients is less diverse compared to healthy controls. Gastrointestinal bacteria may invade the sterile inner colonic adherent mucus layer, disrupt epithelial architecture, and allow leakage of bacterial antigen into the systemic circulation to induce and perpetuate inflammation (8890). A "gut-joint axis" has been proposed where immune cells traffic between the two domains $(91,92)$. Fecal microbiota transplantation (FMT) has shown promising results in the treatment of UC in a Cochrane Database systematic review (93). Positive clinical outcomes are associated with higher dosage and delivery of FMT via lower gastrointestinal tract (94), and may be dependent on stool donor (95). However, a recent RCT on FMT in 31 patients with active PsA randomized to FMT vs. sham treatment was not efficacious for arthritis (96). Further study is required.

In patients with IBD, the number of IL-17-secreting MAIT cells (97), was increased in the gastrointestinal tract as compared to the peripheral blood, echoing PsA studies showing depleted MAIT cells in blood, and increased MAIT cells in inflamed synovia and psoriatic skin $(67,68) \cdot \gamma \delta$ T cells are found in colonic mucosa and represent around $40 \%$ of intraepithelial lymphocytes (98). In contrast to PsA, the presence of $\gamma \delta \mathrm{T}$ cells appears to be protective and anti-inflammatory in patients with IBD. Different subtypes of $\gamma \delta$ T cells may behave differently in different cytokine environments, explaining the diverse observations of $\gamma \delta \mathrm{T}$ cells in PsA and IBD $(99,100)$. As with PsA, Th17 cells are major players in IBD (101). The chemokine receptor CCR6 is the main surface marker of the Th17 lineage. CCL20, a ligand for CCR6, is elevated in IBD gut epithelium and likely contributes to recruitment of CCR6+ type 3 ILC, Th17, and dendritic cells $(102,103)$. Due to high levels of IL-17 and IL-23 in IBD gut epithelia, the IL23/IL-17 pathway was thought to be a therapeutic target (104106). However IL-23 inhibition showed efficacy in patients with IBD but IL-17 inhibition lead to disease exacerbation (107). A possible explanation for this paradox is that IL-17 plays a role in maintaining intestinal barrier and microbial defense (108-110).

\section{Pathogenesis of Uveitis}

The HLA-B*27 is associated with increased risk of AAU (111), and is a common risk locus for PsA (and other SpA) and uveitis (112). HLA haplotypes such as HLA-A*02 (113), HLA-DRB1*08-03 (114), HLA-B*58 (115) were also associated with development of uveitis. Other non-HLA susceptibility loci are major histocompatibility complex class I chain-related gene A (MICA) (116, 117), IL-10 (118), TNF (119), killer immunoglobulin receptor (KIR) (120), and polymorphisms in $I L-23 R$, which all participate in immune response (121). Nonetheless, positive risk polymorphisms do not necessarily translate to uveitis. Other environmental and undiscovered factors are likely required to initiate uveitis in patients with SpA.

The eye is an immunologically privileged organ with a local inhibitory microenvironment, entailing immune ignorance and tolerance to prevent inflammation. The blood-retina barrier and absence of efferent lymphatics reduces exposure of the eye to the circulating immune system (122). In uveitis, infiltration of immune cells into the eye and disrupts the immunologically quiescent environment. However, the trigger of this infiltration is undetermined (123). Some evidence implicates the perturbation of the gut microbiome to SpA-associated uveitis. Animal studies demonstrated trafficking of leucocytes from intestine to eye, supporting the concept of a gut-eye axis (124). Further evidence from retina-specific TCR transgenic mice reared under germfree conditions showed that the severity of uveitis was reduced in the absence of gut microbiota. This reduction of severity was associated with a reduction in Th17 cells in the lamina propria of the intestine. Reconstitution of gut microbiota increased retinaspecific T cell signaling (125). McGonagle et. al (2015) proposed that anterior uveal structures are analogous to entheses due to their mechanical and structural roles in lens suspension. The repeated contractions and relaxations of these structures expose them to mechanical stress just like musculoskeletal entheses, thus providing the initial stimuli for inflammation (126). Like entheseal mesenchymal cells in enthesitis, cells in ciliary body express IL-23R, suggesting receptiveness to IL-23 signaling (127). In patients with uveitis, serum IL-17A levels were elevated during active disease (128). Association between Th17 and the development of uveitis has been observed in animal and clinical studies highlighting the importance of the IL-23/IL-17 axis in driving inflammation in PsA and uveitis (129-132). However, clinical trials have yet to demonstrate the efficacy of IL-17 inhibition in uveitis (133).

\section{MANAGEMENT OF EXTRA-ARTICULAR MANIFESTATIONS IN PSA}

Therapeutic advances in the last decade for PsA and PsO have improved quality of life of many. The European League Against Rheumatism (EULAR) developed algorithm treatment recommendations for the musculoskeletal manifestations of PsA (134). However, patients who have co-existing nonmusculoskeletal manifestations such as IBD and uveitis pose a clinical challenge. The Group for Research and Assessment of Psoriasis and Psoriatic Arthritis (GRAPPA) recommendation guideline highlighted a domain-driven approach focused on peripheral, axial, dactylitis, enthesitis, skin and nails $(135,136)$. The evidence for optimal treatment options for extra-articular manifestations in PsA is lacking and relies on evidence built in the fields of IBD and uveitis as independent conditions. Regardless of domains, the treatment goals are moving toward achieving low or minimal disease activity. Although some treatment options are common across the different domains, the doses may be different. 
TABLE 1 | Therapeutic options and common dosing regimen for PSD and extra-articular manifestations.

\begin{tabular}{|c|c|c|c|c|c|c|}
\hline \multirow[t]{2}{*}{ Drug class } & \multirow[t]{2}{*}{ Agents } & \multicolumn{5}{|c|}{ Dosage for domains } \\
\hline & & Peripheral arthritis & Axial arthritis & Psoriasis & IBD & Uveitis \\
\hline Corticosteroid & & $\begin{array}{l}\text { - Intra-articular corticosteroid } \\
\text { injection as indicated } \\
\text { - Systemic corticosteroid to be } \\
\text { avoided }\end{array}$ & - No indication & $\begin{array}{l}\text { - Topical corticosteroid } \\
\text { - Systemic corticosteroid to be } \\
\text { avoided }\end{array}$ & $\begin{array}{l}\text { - Induction: corticosteroid } \\
\text { short tapering course } \\
\text { - Maintenance: not indicated }\end{array}$ & $\begin{array}{l}\text { - Corticosteroid eye drops } \\
\text { tapering course } \\
\text { - Periocular corticosteroid } \\
\text { injections or intravitreal } \\
\text { implants } \\
\text { - Systemic corticosteroid for } \\
\text { sight-threatening disease }\end{array}$ \\
\hline Immune-modulator & $\begin{array}{l}\text { Methotrexate } \\
\text { Sulfasalazine } \\
\text { Leflunomide } \\
\text { Cyclosporin } \\
\text { Thiopurines }\end{array}$ & $\begin{array}{l}\text { - MTX 10-25 mg QW, PO } \\
\text { - SSZ } 500 \text { mg-3g/day, PO } \\
\text { - LEF 10-20 mg OM, PO } \\
\text { - CyA 2.5-4 mg/kg/day, PO }\end{array}$ & - Not effective & $\begin{array}{l}\text { - MTX } 10-25 \mathrm{mg} \text { QW, PO } \\
\text { - CyA } 2.5-5 \mathrm{mg} / \mathrm{kg} / \text { day, PO }\end{array}$ & $\begin{array}{l}\text { - } 5 \text {-ASA (UC): 1.5-4.5 g/day, } \\
\text { PO } \\
\text { - MTX } 25 \mathrm{mg} \text { QW, SC or IM } \\
\text { - SSZ 3-4 g/day, PO } \\
\text { - AZA } 2.5 \mathrm{mg} / \mathrm{kg}, \text { PO }\end{array}$ & $\begin{array}{l}\text { - MTX 7.5-20 mg QW, PO } \\
\text { - SSZ 3-4 g/day, PO }\end{array}$ \\
\hline \multirow[t]{5}{*}{ TNFI } & Infliximab & $\begin{array}{l}\text { - 5-10 mg/kg loading at W0, } 2 \\
\text { and 6, IV } \\
\text { - then Q6-8W, IV }\end{array}$ & $\begin{array}{l}\text { - 5-10 } \mathrm{mg} / \mathrm{kg} \text { loading at WO, } 2 \\
\text { and 6, IV } \\
\text { - then Q6-8W, IV }\end{array}$ & $\begin{array}{l}\text { - 5-10 mg/kg loading at WO, } 2 \\
\text { and 6, IV } \\
\text { - then Q8W, IV }\end{array}$ & $\begin{array}{l}\text { - Induction (CD/UC): 5-10 } \\
\text { mg/kg loading at WO, } 2 \text { and } \\
\text { 6, IV } \\
\text { - Maintenance (CD/UC): 5-10 } \\
\text { mg/kg Q8W, IV }\end{array}$ & $\begin{array}{l}\text { Off label use } \\
\text { - Induction: } 4 \text { to } 6 \mathrm{mg} / \mathrm{kg} \text { at } \\
\text { W0, 2, 6, then Q4W until } \\
\text { clinical remission, IV } \\
\text { - Maintenance: } 5 \mathrm{mg} / \mathrm{kg} \\
\text { Q10-12W, IV }\end{array}$ \\
\hline & Adalimumab & - $40 \mathrm{mg}$ Q2W, SC & - $40 \mathrm{mg}$ Q2W, SC & - $40 \mathrm{mg}$ Q2W, SC & $\begin{array}{l}-160 \mathrm{mg} \text { or } 80 \mathrm{mg} \text { at } \mathrm{WO} \text {, then } \\
80 \mathrm{mg} \text { at W2, then } 40 \mathrm{mg} \\
\text { Q2W, SC }\end{array}$ & - $40 \mathrm{mg}$ Q2W, SC \\
\hline & Etanercept & - $50 \mathrm{mg}$ QW to BIW, SC* & - $50 \mathrm{mg}$ QW, SC & $\begin{array}{l}\text { - } 50 \mathrm{mg} \text { BIW for } 3 \text { months, } \\
\text { - then } 50 \mathrm{mg} \mathrm{QW}, \mathrm{SC}^{\star}\end{array}$ & $-25 \mathrm{mg}$ BIW, SC & - No indication \\
\hline & Golimumab & $\begin{array}{l}\text { - } 50 \mathrm{mg} \text { Q4W, SC } \\
\text { - Or } 100 \mathrm{mg} \text { Q4W, SC if BW } \\
>100 \mathrm{~kg} \\
\text { - Alternative IV formulation at } \\
2 \mathrm{mg} / \mathrm{kg} \text { at WO and W4, then } \\
\text { Q8W }\end{array}$ & - $50 \mathrm{mg}$ Q4W, SC & $\begin{array}{l}\text { Off label use } \\
\text { - Not primary approved for } \\
\text { Psoriasis }\end{array}$ & $\begin{array}{l}\text { - Induction (CD/UC): } 200 \text { mg } \\
\text { at WO, then } 100 \text { mg at W2 } \\
\text { - Maintenance (CD/UC): } 100 \\
\text { mg Q4W }\end{array}$ & - Off label use \\
\hline & Certolizumab & $\begin{array}{l}\text { - } 400 \text { mg at W0, } 2 \text { and 4, then } \\
200 \text { mg Q2W, SC } \\
\text { - } 400 \text { mg Q4W, SC can be } \\
\text { considered for maintenance }\end{array}$ & $\begin{array}{l}\text { - } 400 \mathrm{mg} \text { at WO, } 2 \text { and 4, then } \\
200 \mathrm{mg} \text { Q2W, SC } \\
\text { - } 400 \mathrm{mg} \text { Q4W, SC can be } \\
\text { considered for maintenance }\end{array}$ & $\begin{array}{l}\text { - } 400 \mathrm{mg} \text { Q2W, SC } \\
\text { - For BW < } 90 \mathrm{~kg} \text {, can } \\
\text { consider } 400 \mathrm{mg} \text { at W0, } 2 \\
\text { and 4, then } 200 \mathrm{mg} \text { Q2W, SC }\end{array}$ & $\begin{array}{l}\text { - Induction (CD/UC): } 400 \mathrm{mg} \\
\text { at WO, 2, 4, SC } \\
\text { - Maintenance (CD/UC): } \\
\quad 400 \mathrm{mg} \text { Q4W, SC }\end{array}$ & $\begin{array}{l}\text { - Ongoing phase III trial, } \\
\text { promising preliminary data } \\
\text { - } 400 \mathrm{mg} \text { at W0, 2, 4; then } \\
200 \mathrm{mg} \text { Q2W }\end{array}$ \\
\hline \multirow[t]{2}{*}{ IL-17i } & Secukinumab & $\begin{array}{l}\text { - Loading } 150 \mathrm{mg} \text { QW for } 5 \\
\text { doses, then monthly, SC } \\
\text { - (300 mg if TNFi experienced) }\end{array}$ & $\begin{array}{l}\text { - Loading } 150 \mathrm{mg} \text { QW for } 5 \\
\text { doses, then monthly, SC }\end{array}$ & $\begin{array}{l}\text { - Loading } 300 \mathrm{mg} \text { QW for } 5 \\
\text { doses, then monthly, SC }\end{array}$ & $\begin{array}{l}\text { - (CD) no difference compared } \\
\text { to placebo, more adverse } \\
\text { events, not indicated }\end{array}$ & $\begin{array}{l}\text { Failure in } 3 \text { RCTs } \\
\text { - Higher dose is superior to } \\
\text { lower doses } \\
\text { - No indication }\end{array}$ \\
\hline & Ixekizumab & $\begin{array}{l}\text { - Loading } 160 \mathrm{mg} \text { once, then } \\
80 \text { mg monthly, SC }\end{array}$ & $\begin{array}{l}\text { - Loading } 160 \text { mg once, then } \\
80 \text { mg monthly, SC }\end{array}$ & $\begin{array}{l}-160 \mathrm{mg} \text { at WO, then } 80 \mathrm{mg} \text { at } \\
\text { W2, 4, 6, 8, 10, and } 12 \text {, then } \\
80 \mathrm{mg} \text { Q4W, SC }\end{array}$ & - No study, no indication & - No study, no indication \\
\hline
\end{tabular}




\section{TABLE 1 | Continued}

\begin{tabular}{|c|c|c|c|c|c|c|}
\hline \multirow[t]{2}{*}{ Drug class } & \multirow[t]{2}{*}{ Agents } & \multicolumn{5}{|c|}{ Dosage for domains } \\
\hline & & Peripheral arthritis & Axial arthritis & Psoriasis & IBD & Uveitis \\
\hline IL-12/23i & Ustekinumab & $\begin{array}{l}\text { - } 45 \mathrm{mg} \text { Q4W for } 2 \text { doses, } \\
\text { then Q12W } \\
\text { - } 90 \mathrm{mg} \text { Q4W for } 2 \text { doses, } \\
\text { then Q12W if } B W>100 \mathrm{~kg}\end{array}$ & - No indication & $\begin{array}{l}\text { - } 45 \mathrm{mg} \text { Q4W for } 2 \text { doses, } \\
\text { then Q12W } \\
\text { - } 90 \mathrm{mg} \text { Q4W for } 2 \text { doses, } \\
\text { then Q12W if } B W>100 \mathrm{~kg}\end{array}$ & $\begin{array}{l}\text { - Induction: single } \\
\text { weight-based dose (<55 kg, } \\
260 \mathrm{mg}, 55-85 \mathrm{~kg}, 390 \mathrm{mg} \text {, } \\
\text { > } 85 \mathrm{~kg} 520 \mathrm{mg} \text { ), IV } \\
\text { - Maintenance: } 90 \mathrm{mg} \text { Q8W, } \\
\text { SC }\end{array}$ & - Ongoing phase 2 trials \\
\hline \multirow[t]{2}{*}{ IL-23i } & Guselkumab & $\begin{array}{l}\text { - Loading } 100 \mathrm{mg} \text { Q4W for } 2 \\
\text { doses, then } 100 \mathrm{mg} \text { Q8W, } \\
\text { SC }\end{array}$ & - No indication & $\begin{array}{l}\text { - Loading } 100 \text { mg Q4W for } 2 \\
\text { doses, then } 100 \text { mg Q8W, } \\
\text { SC }\end{array}$ & - Ongoing phase II/III RCTs & - No study, no indication \\
\hline & Risankizumab & $\begin{array}{l}\text { - } 150 \mathrm{mg} \text { Q4W for } 2 \text { doses, } \\
\text { then Q12W, SC }\end{array}$ & $\begin{array}{l}\text { - No difference compared to } \\
\text { placebo, no indicated }\end{array}$ & $\begin{array}{l}\text { - } 150 \mathrm{mg} \text { Q4W for } 2 \text { doses, } \\
\text { then Q12W, SC }\end{array}$ & $\begin{array}{l}\text { - Ongoing phase III studies in } \\
\text { CD } \\
\text { - Induction (CD): } 600 \mathrm{mg} \text { or } \\
1200 \mathrm{mg} \text { once, IV } \\
\text { - Maintenance (CD): } 600 \mathrm{mg} \\
\text { or } 1200 \mathrm{mg} \text { Q12W, SC }\end{array}$ & - No study, no indication \\
\hline$\alpha 4 \beta 7$ integrin inhibitor & Vedolizumab & - No indication & - No indication & - No indication & $\begin{array}{l}\text { - Induction (CD/UC): } 300 \mathrm{mg} \\
\text { at WO, 2, and 6, IV } \\
\text { - Maintenance (CD/UC): } \\
\text { 300 mg Q8W, IV }\end{array}$ & - No indication \\
\hline \multirow[t]{2}{*}{ JAKi } & Tofacitinib & - 5 mg BD, PO & - 5 mg BD, PO & - $10 \mathrm{mg} \mathrm{BD}, \mathrm{PO}$ & $\begin{array}{l}\text { - Induction (UC only): } 10 \mathrm{mg} \\
\text { BD for at least } 8 \text { weeks; PO } \\
\text { - Maintenance (UC only): then } \\
5 \text { or } 10 \text { mg BD, PO } \\
\text { - CD: No difference compared } \\
\text { to placebo, } \\
\text { - No indication }\end{array}$ & - No study, no indication \\
\hline & Upadacitinib & - 15 mg OM, PO & - 15 mg OM, PO & - 15 mg OM, PO & $\begin{array}{l}\text { - Phase II dose ranging } \mathrm{RCT} \text { in } \\
\mathrm{CD}\end{array}$ & - No study, no indication \\
\hline
\end{tabular}

"Less favored due to lower efficacy; not yet approved by authorities; 5-ASA, 5-aminosalicylic acid; AZA, azathioprine; BD, two times per day; BIW, twice per week; BW, body weight; CD, Crohn's disease; CyA, cyclosporin A; IL, interleukin: IM, intramuscular; IV, intravenous; OM, daily; PO, per oral; JAKi, Janus kinase inhibitors; LEF, leflunomide; MTX, methotrexate; Q, every; SC, subcutaneous; SSZ, sulfasalazine; TNFi, tumor necrosis factor inhibitors; UC, ulcerative colitis; $W$, week. 
We summarize the usual doses used for various domains in Table 1.

\section{Therapeutic Goals}

The treatment targets for patients with IBD are clinical remission, mucosal healing, and restoring quality of life $(137,138)$. The importance of mucosal healing defined as restitution of the intestinal lining and regression or disappearance of endoscopic lesions has been emphasized. Achievement of this target is associated with reduced risk of relapse, reduced hospitalization rates, steroid-free remission, and resection-free status (139-141). With medical advancements, the need for bowel resection is substantially reduced (142).

\section{Medical Therapies for CD}

Corticosteroids can be used to induce clinical remission. It is given either topically as ileal-release budesonide for active mild-to-moderate $\mathrm{CD}$ or systemically for moderateto-severe CD (132). However, systemic corticosteroid should not be used for maintenance $(143,144)$. Early initiation of corticosteroid-sparing immunomodulators such as azathioprine (AZA), mercaptopurine or methotrexate (MTX) for maintenance should be considered, although the level of evidence supporting efficacy of these drugs is relatively low $(144,145)$.

Monoclonal antibody targeting TNF $\alpha$ (TNF inhibitors, TNFi) has become the standard of care for patients with moderateto-severe, active CD. Infliximab (IFX), adalimumab (ADA), and certolizumab (CZP) have demonstrated efficacy in inducing remission and maintenance in RCTs, and well supported by meta-analyses $(146,147)$. We summarized the major RCTs supporting the efficacies of TNFi in IBD Table 2. In a Cochrane Database Systematic review, CD patients who responded to induction by TNFi were more likely to maintain remission at 52 weeks with TNFi compared to placebo (147). Continued treatment with TNFi reduces surgery and hospitalization for CD $(168,169)$. Combination therapy of IFX with AZA was more efficacious than either agent alone in achieving response, inducing clinical and histological remission (156), suggesting synergistic effect. TNFi appears to be more effective when given at earlier stage of disease, with higher rates of response and remission, than given at later stage of disease (170, 171). Early escalation to TNFi treatment should be considered for patients with extensive disease and poor prognostic factors $(144,145)$.

Vedolizumab (VZD) is a monoclonal antibody targeting $\alpha 4 \beta 7$ integrin, which reduces lymphocytes trafficking to the gastrointestinal tract by blocking lymphocyte surface $\alpha 4 \beta 7$ binding to the mucosal addressin cell adhesion molecule1 (MAdCAM-1). The efficacies of VZD in induction and maintenance in CD have been demonstrated in the GEMINI-2 (172) and GEMINI-3 trials (173) (Table 1). In a meta-analysis involving 1716 patients with CD, VZD was more effective than placebo for inducing clinical remission (RR 1.71 [95\% confidence interval, CI: $1.25,2.34], p=0.0008)$, and maintaining clinical remission (RR 1.75 (95\% CI: 1.25, 2.44), $p<0.001$ ).

Ustekinumab (UST) is an antibody targeting the IL-12/23 p40 subunit. The efficacy of UST in inducing remission in $\mathrm{CD}$ has been shown in UNITI-1 and UNITI-2 trials in patients with inadequate response to $\mathrm{TNFi}$, and without prior TNFi failure, respectively. Responders from both studies were randomized to the IM-UNITI maintenance study and demonstrated significantly higher clinical remission rates [high dose: $53 \%, P=0.005$; low dose: $49 \%, P=0.04)$ ] compared to placebo (36\%) at week 44 (162). There is no head-to-head study comparing efficacies between TNFi, VZD and UST. The choice of biological treatment is a shared decision between clinician and patient, and according to the individual risk-benefit preferences.

Risankizumab (RZB), an IL-23/p19 inhibitor met the primary remission induction endpoints in CD in two phase III RCTs, ADVANCE (NCT03105128) and MOTIVATE (NCT03104413) (174). Patients in remission from ADVANCE and MOTIVATE were recruited to the Phase III open-label maintenance study, FORTIFY, showing RZB $360 \mathrm{mg}$ every 8 weeks achieved the coprimary endpoints of clinical remission and endoscopic response at 52 weeks (175).

Blocking IL-17, however, has not been effective in CD. In a phase II trial evaluating safety and efficacy of brodalumab (BRO), a monoclonal antibody targeting IL-17 receptor, the primary induction endpoint was not met. The trial was terminated early due to a disproportionate number of cases of worsening CD (160). In a phase II RCT, two doses of $10 \mathrm{mg} / \mathrm{kg}$ secukinumab (SEC) given intravenously on days 0 and 22, failed to meet the primary endpoint and had more adverse events compared to placebo (176). However, the use IL-17i is not associated with increased incidence of IBD. Data from the SEC development program pooling 7,355 patients with a cumulative exposure of 16,227 person-years of patients exposed to SEC for PsO, PsA or $\mathrm{SpA}$, no increase in exposure adjusted incidence rates of IBD was observed (15). Similarly, events of IBD remained low in the ixekizumab development program that pooled data from 15 RCTs in PsO and PsA (177).

Phase II RCT results for the Janus kinase inhibitor (JAKi), upadacitinib (UPA), in CD are promising. Endoscopic but not clinical remission increased with dose during the induction period (167). However, in a phase II trial for JAKi, tofacitinib (TOF), no statistically significant differences in clinical responses between TOF and placebo were observed at week 4 (164) (Table 2).

\section{Medical Treatment for UC}

Oral 5-ASA (5-aminosalicylic acid) is the standard therapy for induction in mild-to-moderately active UC. For those failing 5ASA or with moderate-to-severe UC, a short 6- to 8-week course of oral corticosteroid is indicated. 5-ASA and thiopurines can be used as maintenance therapy. Like the treatment strategy for $\mathrm{CD}$, early escalation to biologic therapies should be considered for those who failed induction therapy with corticosteroid, or failed maintenance with immunomodulators, and those with poor prognostic factors. TNFi [IFX, ADA, golimumab (GOL)], $\alpha 4 \beta 7$ integrin inhibitor (VZD) and IL12/23i (UST) are approved treatments for induction and maintenance of UC (Table 3). A combination of TNFi with an immunomodulator is more effective. In the UC-SUCCESS trial, patients treated with IFX and AZA were more likely to achieve corticosteroid-free remission at 16 weeks than those receiving either monotherapy (181). 
TABLE 2 | Evidence from major clinical trials for class of therapeutic options for Crohn's disease.

\begin{tabular}{|c|c|c|c|c|c|c|c|}
\hline Class of drug & Agent & $\begin{array}{l}\text { Trial } \\
\text { acronyms }\end{array}$ & RCT Phase & Sample size & Patient population & Treatment vs. comparison & Outcomes \\
\hline \multirow[t]{6}{*}{ TNFi } & ADA & CLASSIC I (148) & $\|$ & 299 & $\begin{array}{l}\text { Active } \mathrm{CD} \text {, naïve to TNFi } \\
\text { (induction) }\end{array}$ & $\begin{array}{l}\text { - ADA } 160 / 80 \mathrm{mg} \\
\text { - Vs. 80/40 mg, } \\
\text { - Vs. 40/20 mg at Wo, 2, SC }\end{array}$ & $\begin{array}{l}\text { - Clinical remission at W4: ADA 160/80 } \\
36 \%(p=0.001), A D A 80 / 4024 \%(p= \\
\text { 0.06), ADA 40/20 18\% ( } p=0.036) \text {, Vs. } \\
\text { PBO 12\% (all comparison vs. PBO). }\end{array}$ \\
\hline & $\mathrm{ADA}$ & CLASSIC II (149) & $\|$ & 276 & $\begin{array}{l}\text { CD achieved induction in CLASSIC I } \\
\text { (maintenance) }\end{array}$ & $\begin{array}{l}\text { Patients achieved remission in CLASSIC I } \\
\text { were re-randomized }(n=55) \text { to } \\
\text { - ADA } 40 \mathrm{mg} \text { QW, SC } \\
\text { - Vs. ADA } 40 \mathrm{mg} \text { Q2W, SC } \\
\text { - Vs. PBO } \\
\text { - Patients not achieved remission } \\
(n=209) \text { received open-labeled ADA } \\
40 \mathrm{mg} \text { Q2W, SC }\end{array}$ & $\begin{array}{l}\text { - Clinical remission at W56 for } \\
\text { re-randomized }(n=55) \text { : } \\
\text { ADA } 40 \mathrm{mg} \text { QW } 83 \%, \text { ADA } 40 \mathrm{mg} \text { Q2W } \\
79 \% \text { Vs. PBO } 44 \% \text { (all } p<0.05 \text { vs. PBO). } \\
\text { - Clinical remission at W56 for open } \\
\text { labeled patients }(n=209) \text { : ADA } 46 \%\end{array}$ \\
\hline & ADA & GAIN (150) & III & 325 & Active CD, TNFi IR (induction) & $\begin{array}{l}\text { ADA } 160 \mathrm{mg} \text { at WO, then } 80 \mathrm{mg} \text { at W2, } \\
\text { SC vs. PBO }\end{array}$ & $\begin{array}{l}\text { - Clinical remission at W4: } \\
\text { ADA } 21.6 \% \text { vs. PBO } 6.7 \%,(p<0.001) \text {. }\end{array}$ \\
\hline & ADA & CHARM (151) & III & 854 & $\begin{array}{l}\text { Active CD despite } \\
\text { immunomodulators, non-TNFi IR. } \\
\text { All received open labeled induction: } \\
\text { ADA } 80 \text { mg at WO, } 40 \text { mg at W2, SC } \\
\text { (maintenance) }\end{array}$ & $\begin{array}{l}\text { Maintenance: } \\
\text { - ADA } 40 \mathrm{mg} \text { Q2W, } \\
\text { - vs. } 40 \mathrm{mg} \text { QW } \\
\text { - vs. PBO }\end{array}$ & $\begin{array}{l}\text { - Clinical remission at W26: } \\
\text { ADA QW 47\% vs. ADA Q2W 40\% vs. PBO } \\
17 \% \text { (all } p<0.001 \text { vs. PBO). } \\
\text { - Clinical remission at W56: } \\
\text { ADA QW } 41 \% \text { vs. ADA Q2W 36\% vs. } \\
\text { PBO } 12 \% \text {, (all } p<0.001 \text { vs. PBO). }\end{array}$ \\
\hline & $\mathrm{ADA}$ & EXTEND (152) & III & 129 & $\begin{array}{l}\text { Active CD, responded to open } \\
\text { labeled ADA induction (160/80 mg at } \\
\text { W0, 2, SC) at W4 } \\
\text { (maintenance) }\end{array}$ & $\begin{array}{l}\text { Maintenance: } \\
\text { - ADA } 40 \mathrm{mg} \text { QW, SC } \\
\text { - vs. } 40 \mathrm{mg} \text { Q2W, SC } \\
\text { - vs. PBO }\end{array}$ & $\begin{array}{l}\text { - Mucosal healing at W12: } \\
\text { ADA 27\% vs. PBO 13\%, }(p=0.056) \text {. } \\
\text { - Clinical remission at W12: } \\
\text { ADA 52\% vs. PBO } 28 \%(p=0.006) \\
\text { - Mucosal healing at W52: } \\
\text { ADA } 24 \% \text { vs. PBO } 0 \%(p<0.001) \text {. } \\
\text { - Clinical remission at W52: } \\
\text { ADA } 28 \% \text { vs. PBO } 3 \%(p<0.001) .\end{array}$ \\
\hline & IFX & (153) & $\|$ & 108 & $\begin{array}{l}\text { Moderate to severe } \mathrm{CD} \text {, naïve to } \mathrm{TNFi} \\
\text { (induction) }\end{array}$ & $\begin{array}{l}\text { - IFX } 5 \text { mg/kg, once, IV } \\
\text { - vs. IFX } 10 \mathrm{mg} / \mathrm{kg} \text {, once, IV } \\
\text { - vs. IFX } 20 \mathrm{mg} / \mathrm{Kg} \text {, once, IV } \\
\text { - vs. PBO }\end{array}$ & $\begin{array}{l}\text { - Clinical response W4: } \\
\text { IFX } 5 \mathrm{mg} 81 \%(p=0.33) \text { vs. } 10 \mathrm{mg} 50 \%(P \\
=0.26 \text { ) vs. } 20 \mathrm{mg}: 64 \%(p=0.01) \text { vs. PBO } \\
\text { 17\%. (all comparison vs. PBO) } \\
\text { - Clinical remission W4: } \\
\text { IFX (all doses) } 33 \% \text { vs. PBO } 4 \% \quad(p \\
=0.005) \text {. } \\
\text { - Clinical response W12: } \\
\text { IFX (all doses) } 41 \% \text { vs. PBO } 12 \% \text { p } \\
=0.008) .\end{array}$ \\
\hline
\end{tabular}


TABLE 2 | Continued

\begin{tabular}{|c|c|c|c|c|c|c|c|}
\hline Class of drug & Agent & $\begin{array}{l}\text { Trial } \\
\text { acronyms }\end{array}$ & RCT Phase & Sample size & Patient population & Treatment vs. comparison & Outcomes \\
\hline & $\mathrm{IFX}$ & ACCENT-I (154) & III & 573 & $\begin{array}{l}\text { Active } \mathrm{CD} \text {, despite } \\
\text { immunomodulators naïve to TNFi, all } \\
\text { received open-labeled IFX induction, } \\
\text { then re-randomized for maintenance } \\
\text { (induction and maintenance) }\end{array}$ & $\begin{array}{l}\text { Open-labeled induction (all): } \\
\text { IFX } 5 \mathrm{mg} / \mathrm{kg} \text { at WO, IV par Randomized at } \\
\text { W2 for maintenance: } \\
\text { - IFX } 10 \mathrm{mg} / \mathrm{kg} \text { at W2, 6, then Q8W, IV } \\
\text { - Vs. IFX } 5 \mathrm{mg} / \mathrm{kg} \text { at W2, 6, then } 5 \mathrm{mg} / \mathrm{kg} \\
\text { Q8W, IV } \\
\text { - Vs. PBO }\end{array}$ & $\begin{array}{l}\text { - Induction: } \\
58 \% \text { responded to initial IFX at W2. } \\
\text { - Clinical remission at W30: } \\
\text { IFX10 mg } / \mathrm{kg} 45 \%(p=0.003) \text {, vs. IFX5 } \\
\mathrm{mg} / \mathrm{kg} 39 \%(p=0.0002) \text {, vs. PBO } 21 \% \text {. } \\
\text { (all comparison vs. PBO) } \\
\text { - Median time to loss of response at W54: } \\
\text { IFX } 10 \mathrm{mg} / \mathrm{kg}>54 \mathrm{~W}(p=0.002) \text {, vs. } \\
\text { IFX } 5 \mathrm{mg} / \mathrm{kg} 38 \mathrm{~W}(p=0.0002) \text {, vs. PBO } \\
19 \mathrm{~W} \text { (all comparison vs. PBO) }\end{array}$ \\
\hline & IFX & ACCENT-II (155) & III & 282 & $\begin{array}{l}\text { Fistulating CD, naïve to TNFi } \\
\text { (induction and maintenance) }\end{array}$ & $\begin{array}{l}\text { Open-labeled induction (all): } \\
\text { IFX } 5 \mathrm{mg} / \mathrm{kg} \text { at W0, 2, 6, IV } \\
\text { - Randomized at W14 for maintenance: } \\
\text { - IFX } 5 \mathrm{mg} / \mathrm{kg} \text { Q } 8 \text { W, IV } \\
\text { - vs. PBO }\end{array}$ & $\begin{array}{l}\text { - Induction: } \\
69 \% \text { responded to initial IFX at W14. } \\
\text { - Time to loss of response: } \\
\text { IFX >40W vs. PBO } 14 W(p<0.001) \\
\text { - Clinical response W54: } \\
\quad \text { IFX } 36 \% \text { vs. PBO } 19 \%(p=0.009)\end{array}$ \\
\hline & $\mathrm{IFX}$ & SONIC (156) & III & 508 & $\begin{array}{l}\text { Active } \mathrm{CD} \text {, naïve to immunomodulator } \\
\text { and TNFi } \\
\text { (induction and maintenance) }\end{array}$ & $\begin{array}{l}\text { IFX } 5 \mathrm{mg} / \mathrm{kg} \text { at W0, 2, 6, then Q8W + AZA } \\
2.5 \mathrm{mg} / \mathrm{kg} / \text { day } \\
\text { - vs. IFX alone } \\
\text { - vs. AZA alone }\end{array}$ & $\begin{array}{l}\text { - Corticosteroid-free remission W26: } \\
\text { IFX+AZA: } 57 \% \text { ( } p=0.002 \text { vs. IFX; } p< \\
0.001 \text { vs. PBO), vs. IFX alone: } 44 \%(p= \\
0.006 \text { vs. AZA), vs. AZA alone: } 30 \% \text {. } \\
\text { - Mucosal healing W26: } \\
\text { IFX+AZA } 44 \%(p=0.06 \text { vs IFX; } p= \\
<0.001 \text { vs. AZA), vs. IFX alone } 30 \%(p= \\
0.02 \text { vs. AZA), vs. AZA alone: } 17 \% .\end{array}$ \\
\hline & CZP & PRECiSE I (157) & III & 662 & $\begin{array}{l}\text { Active CD, } 17 \% \text { concomitant } \\
\text { corticosteroid and } \\
\text { immunomodulators, 28\% prior TNFi } \\
\text { (induction) }\end{array}$ & $\begin{array}{l}\text { - CZP } 400 \text { mg at WO, 2, 4, then Q4W, SC } \\
\text { - vs. PBO }\end{array}$ & $\begin{array}{l}\text { - Clinical response at W6: } \\
\text { CZP } 35 \% \text { vs. PBO } 27 \%,(p=0.02) ; \\
\text { - Clinical response at both W6 and W26: } \\
\text { CZP } 23 \% \text { vs. PBO } 16 \%,(p=0.02) \\
\text { - Remission at W6: } \\
\text { CZP } 14 \% \text { vs. PBO } 10 \%(p=0.17) \\
\text { - Remission at both W6 and W26: } \\
\text { CZP } 22 \% \text { vs. PBO } 17 \%(p=0.07)\end{array}$ \\
\hline & & PRECiSE II (158) & III & 428 & $\begin{array}{l}\text { Active CD, } 24 \% \text { concomitant } \\
\text { corticosteroid and } \\
\text { immunomodulators, 15\% prior TNFi } \\
\text { (maintenance) }\end{array}$ & $\begin{array}{l}\text { Open-labeled induction }(n=668) \text { : } \\
\text { CZP } 400 \text { mg at WO, } 2,4, \text { SC } \\
\text { Patient with clinical response were } \\
\text { randomized at W6 for maintenance } \\
(n=428) \text { : } \\
\text { - CZP } 400 \text { mg Q4W, SC } \\
\text { - vs. PBO }\end{array}$ & $\begin{array}{l}\text { - Clinical response at induction (W6): 64\% } \\
\text { - Clinical remission at W26: CZP 48\% vs. } \\
\text { PBO 29\% ( } p<0.001) \text {. }\end{array}$ \\
\hline
\end{tabular}


Active CD, TNFi IR

(maintenance)

Open-labeled induction $(n=539)$

CZP $400 \mathrm{mg}$ at WO, 2, 4, SC

- Patient with clinical response were

randomized at W6 for open-labeled

maintenance $(n=329)$

CZP $400 \mathrm{mg}$ Q2W, SC

- vs. CZP $400 \mathrm{mg}$ Q4W, SC

- vs. PBO

Active CD, TNFi IR (induction and maintenance)

\section{Induction W0-8 $(n=539)$ : \\ UST 1, 3, 6 mg/kg, SC}

- vs. PBO

- Maintenance 8-36W (re-randomized at W8):

UST $90 \mathrm{mg}$ at W8 and 16, SC - vs. PBO

UNITI-1: active CD, TNFi IR $(n=741)$. Induction WO-8 (UNITI-1 or 2): UNITI-2: active CD, UST $130 \mathrm{mg}$, SC immunomodulator IR $(n=628)$. - vs. UST $6 \mathrm{mg} / \mathrm{kg}$, SC IM-UNITI: Who had clinical response in UNITI- 1 and $2(n=397)$
- vs. PBO

- Maintenance W8-44:

- UST 90 mg/8W, SC

- vs. UST $90 \mathrm{mg} / 12 \mathrm{~W}, \mathrm{SC}$

- vs. PBO
- Clinical response at induction (W6): 62\%

- Clinical response at W26: CZP Q2W 37\%

vs. CZP Q4W 40\% ( $P=0.55)$.

Clinical remission at W26: CZP Q2W

$30 \%$ vs. CZP Q4W 29\% ( $p=0.81$ ).

Early termination due to worsening $C D$ in active treatment groups, $n=130$ analyzed at termination

- Clinical response at W6: BRO $210 \mathrm{mg}$ $16 \%$ vs. $350 \mathrm{mg} 27 \%$ vs. $700 \mathrm{mg} 15 \%$ vs. PBO $13 \%$.

- Clinical remission at W6: BRO $210 \mathrm{mg}$ $3 \%$ vs. $350 \mathrm{mg} 15 \%$ vs. $700 \mathrm{mg} 9 \%$ vs. PBO 13\%.

- Clinical remission at W6 (induction): UST6 mg/kg $39.7 \%$ vs. PBO $23.5 \%$ (p $=0.005)$ NS for other UST doses

Maintenance for those responded to induction, $n=145$

- Clinical response at W22: UST 69.4\% vs. PBO $42.5 \%(p<0.05)$

- Clinical remission at W22: UST 42\% vs. PBO 27\% ( $p<0.05$ )

Induction: Clinical remission at W8:

- UNITI-1 UST 6 mg/kg 38\% $(p<0.001)$ vs. UST $130 \mathrm{mg} 34 \%$ ( $p=0.001)$ vs. PBO $20 \%$ (all comparison vs. PBO)

- UNITI-2 UST 6 mg/kg 58\% $(p<0.001)$ vs. UST $130 \mathrm{mg}: 47 \%(p=0.001)$ vs. PBO 32\% (all comparison vs. PBO)

Maintenance (IM-UNITI)

- Clinical response: UST $90 \mathrm{mg}$ Q8W 59\% $(p=0.02)$ vs. UST $90 \mathrm{mg}$ Q12W $58 \%$ $(p=0.03$ ) vs. PBO $44 \%$ (all comparison vs. PBO)

- Clinical remission UST $90 \mathrm{mg}$ Q8W 53\% $(p=0.02)$ vs. UST $90 \mathrm{mg}$ Q12W 49\% ( $p$ $=0.03$ ) vs. $\mathrm{PBO} 36 \%$ (all comparison vs. $\mathrm{PBO}$ ) 


L-23i- $=0$

(163)

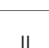

121

Active CD, TNFi IR

(induction)

- RZB $600 \mathrm{mg}$ Q4W

-vs. RZB $200 \mathrm{mg}$ Q4W

- vs. PBO

TOF 15, 5, $5 \mathrm{mg}$ BD, PO vs. $\mathrm{PBO}$

Active CD (induction)

Active CD, \% prior TNFi (induction and maintenance)

280
(induction)
180
(maintenance)

Active CD, 27\% prior bowel resection, 58\% prior TNFi (induction)

220 -vs. TOF 5 mg BD, PO
Active $\mathrm{CD}$ (induction and maintenance)
Induction $0-8 \mathrm{~W}(n=280)$

- TOF $10 \mathrm{mg}$ BD, PO

Maintenance 8-26W for those

responded to TOF induction $(n=180)$ :

TOF $10 \mathrm{mg}$ BD, PO

vs. TOF 5 mg BD, PO

- vs. PBO

FIL 200 mg/day, PO

vs. $\mathrm{PBO}$

Induction WO-16:

UPD 3, 6, 12, $24 \mathrm{mg} \mathrm{BD}$ or $24 \mathrm{mg} /$ day vs. PBO

Maintenance W16-52

UPD 3, 6, 12, $24 \mathrm{mg}$ BD or $24 \mathrm{mg} /$ day

No PBO control
- Clinical response at W12: RZD $600 \mathrm{mg}$ $42 \%(p=0.0366)$ vs. RZD $200 \mathrm{mg} \mathrm{37 \%}$ $(p=N S)$ vs. PBO $21 \%$ (all comparison vs. PBO)

Clinical remission at W12: RZD $600 \mathrm{mg}$ $37 \%(p=0.025)$ vs. RZD $200 \mathrm{mg}: 24 \%$ $(p=N S)$ vs. PBO $15 \%(p=0.049)$ (all. comparison vs. $\mathrm{PBO}$ )

- Clinical response W4:

TOF $15 \mathrm{mg} 46 \%(p=0.467)$ vs. $5 \mathrm{mg} 58 \%$ $(p=0.466)$ vs. $5 \mathrm{mg}: 36 \%(p \geq 0.999)$ vs. PBO 47\% (all comparison vs. PBO).

Clinical remission at W4:

TOF $15 \mathrm{mg} 14 \%(p=0.540)$ vs. $5 \mathrm{mg} 24 \%$ $(p=0.776)$ vs. $5 \mathrm{mg} 31 \%(p=0.417)$ vs. PBO 21\% (all comparison vs. PBO).

- Clinical remission at W8 (induction): TOF $10 \mathrm{mg} 43 \%(p=0.392)$ vs. TOF $5 \mathrm{mg} 44 \%$ ( $p=0.325$ ) vs. PBO $36.7 \%$ (all comparison vs. PBO).

- Clinical remission at W26 (maintenance): TOF $10 \mathrm{mg} 56 \%(p=0.13)$ vs. TOF $5 \mathrm{mg}$ $40 \%(p=N S)$ vs. PBO $38.1 \%$. (all comparison vs. $\mathrm{PBO}$ )

- Clinical remission at W10:

FIL $47 \%$ vs. PBO $36.7 \%$. ( $p=0.0077)$

Clinical remission at W16:

UPA $3 \mathrm{mg} 13 \%$ (NS) vs. $6 \mathrm{mg} 27 \%$ (p $<0.1$ vs. PBO) vs. $12 \mathrm{mg} 11 \%$ (NS) vs. $24 \mathrm{mg}$ : $22 \%$ (NS) vs. $24 \mathrm{mg} /$ day $14 \%$ (NS) vs. PBO

$11 \%$. (all comparison vs. PBO)

Endoscopic remission at W16:

- UPA $3 \mathrm{mg} 10 \%(p<0.1)$ vs. $6 \mathrm{mg} 8 \%$ (NS) vs. $12 \mathrm{mg} 8 \%(p<0.1)$ vs. $24 \mathrm{mg}$ $22 \%(p<0.01)$ vs. $24 \mathrm{mg} /$ day $14 \%(p$ $<0.05$ ) vs. PBO $0 \%$. (all comparison vs. PBO).

- Maintenance: Efficacy

was maintained for most endpoints through week 52

ADA, adalimumab; AZA, azathioprine; BD, Twice daily; BRO, brodalumab; BW, Body weight; CD, Crohn's Disease; CZP, certolizumab; FIL, filgotinib; Gp, Group; IFX, infliximab; IL, Interleukin; i, inhibitor; IR, inadequate responder; IV, intravenous; JAKi, Janus kinase inhibitor; NS, not statistically significant; PBO, placebo; Q, every; RCT, Randomized control trial; RZB, risankizumab; TNF, tumor necrosis factor; inhibitor; TOF, tofacitinib; UPA, upadacitinib; UST, ustekinumab; vs., versus; W, week; yr, year. 
In a head-to-head study (VARSITY) comparing TNFi and VZD in patients with moderate-to-severe UC, 769 patients with moderate-to-severe active UC were randomized to receive VDZ or ADA (185). Only $26 \%$ of patients in either group were on concomitant immunomodulators. At week 52, a higher percentage of patients achieved clinical remission ( $31.3 \mathrm{vs.} 22.5 \%$; $P=0.006$ ), and endoscopic improvement (39.7 vs. $27.7 \% ; P<$ 0.001 ) in VDZ compared to ADA group. Whilst corticosteroidfree clinical remission occurred at a higher rate in the group receiving ADA compared to $\mathrm{VDZ}$ (21.8 vs. $12.6 \%$; difference, -9.3 percentage points; $95 \% \mathrm{CI},-18.9$ to 0.4 ). Despite a slight superiority of VDZ over ADA, more data are pending for consistency and class effect. The choice of biologics is, again, a shared decision between between clinician and patient.

Due to the ineffectiveness of IL-17i for CD, there have not been trials for their use in UC. As for IL-23i, there is an ongoing phase II/III trial of RZB for UC (NCT03398148).

In contrast to $\mathrm{CD}$, the JAKi, TOF, was approved for use in moderate-to-severe UC based on three pivotal phase III OCTAVE studies, showing a significantly greater percentage of clinical remission at week 8 for induction, and remission at week 52 for maintenance in TOF compared to placebo group (186). UPA met the clinical remission, endoscopic improvement and histological improvement endpoints in a phase III induction trial for moderate-to-severe UC (187).

\section{Medical Treatment for Uveitis}

Prompt control of inflammation using topical corticosteroid is the first-line treatment for anterior uveitis in SpA. Typically, prednisolone acetate $1 \%$ eye drops are used as for severe AAU while milder corticosteroids such as dexamethasone $0.1 \%$ may be used for maintenance. A mydriatic drug is often prescribed together to reduce the development of posterior synechia and reduce pain from ciliary body spasm. Periocular corticosteroid injections or intravitreal implants can be used for more chronic cases. Adverse effects of corticosteroid in the eyes include cataract and ocular hypertension in up to $30 \%$ of patients. Oral corticosteroids may be used for acute management of severe and sight-threatening posterior uveitis such as vasculitis and cystoid macular edema, however, immunotherapy should be considered early in these cases to reduce recurrences (188). Traditional immunomodulators such as sulfasalazine (SSZ) and MTX may be tried although few data have supported their efficacy. Monoclonal antibody-TNFi including IFX, ADA, GOL and CZP are considered as effective treatment options for both acute flares and reducing recurrences of AAU (189). We summarize the major RCTs of therapeutic options for uveitis in Table 4. In a post-hoc analysis pooling data from four RCTs with TNFi in AS, the frequency of AAU flares was substantially lower among IFX or etanercept (ETN) treated than placebo treated patients. Lower frequency of AAU flares was seen in the open-labeled extension phase compared to the placebo phase of the trial (TNFi: 6.8 flares per 100 patient-years compared to PBO: 15.6 per 100 patient-years, $p=0.01$ ) (198). ADA is the only TNFi licensed for treatment of non-infectious uveitis in adult following favorable results in 2 phase III RCTs. In the VISUAL I study, patients with active non-infectious intermediate, posterior uveitis or panuveitis were randomized to receive ADA or placebo after a prednisolone burst $(60 \mathrm{mg})$ with tapering course. Patients treated with ADA were less likely than those treated with placebo to have treatment failure (hazard ratio, $\mathrm{HR}, 0.50 ; 95 \% \mathrm{CI}, 0.36$ to $0.70 ; P<0.001$ ). The VISUAL II study recruited 226 patients with inactive, noninfectious intermediate, posterior, or panuveitis controlled by $10-$ $35 \mathrm{mg}$ /day of prednisone were randomized to ADA vs. placebo. All patients underwent a mandatory prednisolone tapering to $0 \mathrm{mg}$ by week 19 . The time to treatment failure was significantly longer in ADA compared to placebo arm (median > 18 months vs. 8.3 months; HR 0.57, 95\% CI 0.39-0.84; $p=0.004$ ) (191). ADA is also licensed for juvenile idiopathic arthritis-related uveitis. In an open-label study in 93 AS patients with history of uveitis, GOL reduced uveitis flares compared to patients' historical control 12month prior to initiation of GOL (192). There is an ongoing phase III 96-week open-label study for CZP in 115 patients with axial $\mathrm{SpA}$ and recurrent uveitis. In the 48 -week interim analysis of 85 patients, uveitis flares were substantially reduced during the CZP treated period compared to the historical rates (64.0 and $31.5 \%$ respectively) (193). The use of ETN in the management of uveitis has diminished in favor of other TNFi because of its weaker ability in preventing flares.

Despite implicated in the pathogenesis of uveitis, inhibiting IL-17A was not effective for uveitis. In three RCTs, SEC failed to meet the primary efficacy endpoints (194). In another RCT comparing three doses of SEC, statistical higher response rates and remission on day 57 for the high dose regimen (30 $\mathrm{mg} / \mathrm{kg}$ intravenously Q2W for 4 doses) was seen compared to the other two lower dose regimens, suggesting a higher dose intravenous regimen may be required to deliver SEC in therapeutic concentrations (195). Results are awaiting for two trials using UST in active sight-threatening uveitis (STAR) (196) and Behçet uveitis (STELABEC) (197), which may provide insight for its potential use in PsA related uveitis.

Minimal data exist for use of JAKi in uveitis. One phase 2 RCT evaluating filgotinib (FIL) in patients with active non-infectious uveitis (NCT03207815) is ongoing.

\section{MANAGEMENT OF PSA WITH CONSIDERATION OF EXTRA-ARTICULAR MANIFESTATIONS}

Given the heterogeneity in manifestations, enhanced collaboration between disciplines are required to deliver optimal care for PsD (199). While collaborations between rheumatologists and dermatologists are increasing (200), collaborations with gastroenterologists and ophthalmologists have traditionally been weaker. Apart from setting up combined clinics, collaborations between disciplines can take other forms as determined by needs and circumstances of different institutions. Minimally, identifying key stakeholders specializing in the care of PsA patients and keeping them in close communication over the management plan is essential. These collaborations serve both clinical and educational needs. Close collaboration between the various disciplines will help in early diagnosis of the various manifestations, providing expert advice on choice of 
TABLE 3 | Evidence from clinical trials for class of therapeutic options for ulcerative colitis.

\begin{tabular}{|c|c|c|c|c|c|c|}
\hline Class of drug & Agent & $\begin{array}{l}\text { Trial } \\
\text { acronyms }\end{array}$ & $\begin{array}{c}\text { RCT } \\
\text { Phase }\end{array}$ & $\begin{array}{l}\text { Sample } \\
\text { size }\end{array}$ & Patient population & Treatment vs. comparison \\
\hline \multirow[t]{3}{*}{ TNFi (mAb) } & $\mathrm{ADA}$ & Ultra 1 (178) & III & 186 & $\begin{array}{l}\text { Active UC, despite corticosteroid } \\
\text { and/or immunomodulators } \\
\text { (induction) }\end{array}$ & $\begin{array}{l}\text { - ADA } 160 \mathrm{mg} \text { at WO, } 80 \mathrm{mg} \text { at W2, } \\
40 \mathrm{mg} \text { at } \mathrm{W} 4 \text { and } 6, \mathrm{SC} \\
\text { - Vs. ADA } 80 \mathrm{mg} \text { at WO and } 2,40 \mathrm{mg} \text { at } \\
\text { W4 and } 6, \mathrm{SC} \\
\text { - Vs. PBO }\end{array}$ \\
\hline & $A D A$ & Ultra 2 (179) & III & 494 & $\begin{array}{l}\text { Active UC, despite corticosteroid } \\
\text { and/or immunomodulators } 40 \% \\
\text { prior TNFi (induction and } \\
\text { maintenance) }\end{array}$ & $\begin{array}{l}\text { - ADA } 160 \mathrm{mg} \text { at WO, } 80 \mathrm{mg} \text { at W2, and } \\
\text { then } 40 \mathrm{mg} \text { Q2W, SC } \\
\text { - Vs. PBO }\end{array}$ \\
\hline & IFX & ACT I (180) & III & 364 & $\begin{array}{l}\text { Active UC despite corticosteroid } \\
\text { and/or thiopurines } \\
\text { (induction and maintenance) }\end{array}$ & $\begin{array}{l}\text { - IFX } 5 \mathrm{mg} \text { or } 10 \mathrm{mg} / \mathrm{kg} \text { at } \mathrm{W} 0,2,6,14 \text {, } \\
22,30,38 \text {, and } 46, \mathrm{IV} \\
\text { - Vs. PBO }\end{array}$ \\
\hline
\end{tabular}

Outcomes

Clinical remission at W8:

ADA160/80 18.5\% ( $p=0.031$ vs. PBO)

vs. ADA $80 / 4010.0 \%$ ( $p=0.833$ vs. $P B O)$

vs. $9.2 \% \mathrm{PBO}$

- Clinical remission at W8:

- ADA 16.5\% vs PBO 9.3\% ( $p=0.019)$

- Clinical remission at W52 ADA $17.3 \%$ vs PBO $8.5 \%(p=0.004)$

- Better response in TNFi naïve patients

- At W8, higher clinical response in IFX groups:

IFX $10 \mathrm{mg} / \mathrm{kg}$ vs. IFX $5 \mathrm{mg} / \mathrm{kg}$ vs. PBO: $61.5 \%$ vs. $69.4 \%$ vs. $37.2 \%$, (all $p<0.001$ compared to $\mathrm{PBO}$ ).

At W8, higher clinical remission in IFX groups:

IFX $10 \mathrm{mg} / \mathrm{kg}$ vs. IFX $5 \mathrm{mg} / \mathrm{kg}$ vs. PBO: $32 \%$ vs. $38.8 \%$ vs. $14.9 \%$, (all $p<0.001$ compared to PBO).

Remission rate at W54:

IFX $10 \mathrm{mg} / \mathrm{kg}$ vs. IFX $5 \mathrm{mg} / \mathrm{kg}$ vs. PBO

(34.4\% vs. $34.7 \%$ vs. $16.5 \%)$, (all $p=$

0.001 compared to PBO).

Active UC despite corticosteroid and/or thiopurines and 5-ASA (induction and maintenance)
IFX $5 \mathrm{mg}$ or $10 \mathrm{mg} / \mathrm{kg}$ at WO, 2, 6, 14 , and 22, IV

- Vs. PBO 


\begin{tabular}{|c|c|c|c|c|c|c|c|}
\hline Class of drug & Agent & $\begin{array}{l}\text { Trial } \\
\text { acronyms }\end{array}$ & $\begin{array}{l}\text { RCT } \\
\text { Phase }\end{array}$ & $\begin{array}{l}\text { Sample } \\
\text { size }\end{array}$ & Patient population & Treatment vs. comparison & Outcomes \\
\hline & IFX & US-SUCCESS (181) & III & $\begin{array}{c}239 \\
\text { (planned 600) }\end{array}$ & $\begin{array}{l}\text { Active UC } \\
\text { (induction) }\end{array}$ & $\begin{array}{l}\text { - IFX } 5 \mathrm{mg} / \mathrm{kg} \text { at weeks } 0,2,6 \text {, and 14, IV } \\
+ \text { AZA } 2.5 \mathrm{mg} / \mathrm{kg} / \text { day, PO } \\
\text { - Vs. IFX alone } \\
\text { - Vs. AZA alone }\end{array}$ & $\begin{array}{l}\text { - Study terminated early before enrolment } \\
\text { target (intermittent IFX regimen raised } \\
\text { concern for injection site reaction in } \\
\text { another study) } \\
\text { - Corticosteroid-free remission at } W 16 \text { : } \\
\text { IFX+AZA } 39.7 \% \text { vs. IFX alone } 22.1 \%(p= \\
0.017 \text { ) vs. AZA alone } 23.7 \%(p=0.032) \text {. } \\
\text { - Mucosal healing at W16: } \\
\text { IFX+AZA } 62.8 \% \text { vs. IFX alone } 54.6 \%(p= \\
0.295 \text { ) vs. AZA alone } 36.8 \%(p=0.001) \text {. }\end{array}$ \\
\hline & GOL & $\begin{array}{l}\text { PURSUIT } \\
\text {-SC (182) }\end{array}$ & III & 761 & $\begin{array}{l}\text { Active UC despite corticosteroid } \\
\text { and/or immunomodulators } \\
\text { (induction) }\end{array}$ & $\begin{array}{l}\text { - GOL } 400 \mathrm{mg} \text { at WO, then } 200 \mathrm{mg} \text { at W2, } \\
\text { SC } \\
\text { - Vs. GOL } 200 \mathrm{mg} \text { at WO, then } 100 \mathrm{mg} \text { at } \\
\text { W2, SC } \\
\text { - Vs PBO }\end{array}$ & $\begin{array}{l}\text { - Clinical response at W6: } \\
\text { GOL } 400 / 20054.9 \% \text { vs. GOL } 200 / 100 \\
51 \% \text { vs. PBO } 30.3 \% \text { (all } p<0.001 \text { vs. PBO) } \\
\text { - Clinical remission at W6: } \\
\text { GOL } 400 / 20017.9 \% \text { vs. GOL } 200 / 100 \\
17.8 \% \text { vs. PBO } 6.4 \% \text { (all } p<0.001 \text { vs. } \\
\text { PBO) } \\
\text { - Mucosal healing at W6: } \\
\text { GOL } 400 / 20045.1 \% \text { vs. GOL } 200 / 100 \\
42.3 \% \text { ( } p=0.0014 \text { vs. PCB) vs. PBO } \\
28.7 \% \text { (all } p<0.001 \text { vs. PBO) }\end{array}$ \\
\hline & GOL & $\begin{array}{l}\text { PURSUIT } \\
\text {-MAINTENANCE } \\
\text { (183) }\end{array}$ & III & 464 & $\begin{array}{l}\text { UC patients responded to GOL } \\
\text { induction } \\
\text { (maintenance) }\end{array}$ & $\begin{array}{l}\text { - GOL } 100 \text { mg Q4W, SC } \\
\text { - Vs. GOL } 50 \text { mg Q4W, SC } \\
\text { - Vs. PBO }\end{array}$ & $\begin{array}{l}\text { - Clinical response maintained at } 54 \text { W: } \\
\text { GOL100 } 49.7 \%(p=0.01 \text { ) vs. GOL50 } 47 \% \\
(p<0.001) \text { vs. } 31.2 \% \text { PBO (all comparison } \\
\text { vs. PBO) } \\
\text { - Clinical remission at both W30 and W54: } \\
\text { GOL100 } 27.8 \%(p=0.004) \text { vs. GOL50 } \\
23.2 \% \text { (NS) vs. } 15.6 \% \text { PBO (all comparison } \\
\text { vs. PBO) } \\
\text { - Mucosal healing at both W30 and W54: } \\
\text { GOL100 } 42.4 \%(p=0.002 \text { ) vs. GOL50 } \\
41.7 \%(p=0.011) \text { vs. } 26.6 \% \text { PBO (all } \\
\text { comparison vs. PBO) }\end{array}$ \\
\hline$\alpha 4 \beta 7$ integrin inhibitor & VDL & GERMIN I (184) & III & $\begin{array}{l}\text { Induction }= \\
\quad 886 \\
\text { Maintenance } \\
\quad=373\end{array}$ & $\begin{array}{l}\text { - Induction: active UC despite } \\
\text { corticosteroid and/or } \\
\text { immunomodulators ( } 48.2 \% \text { prior } \\
\text { TNFi) } \\
\text { maintenance: patients responded to } \\
\text { induction phase }\end{array}$ & $\begin{array}{l}\text { - VDL } 300 \text { mg Q4W, IV } \\
\text { - Vs. VDL } 300 \text { mg Q8W, IV } \\
\text { - Vs. PBO } \\
\text { - (both induction and maintenance) }\end{array}$ & $\begin{array}{l}\text { - Induction phase at W6: } \\
\text { - Clinical response: VDL } 47 \% \text { vs. PBO } \\
25.5 \%, p<0.001 \\
\text { - Clinical remission: VDL } 16.9 \% \text { vs. PBO } \\
5.4 \%, p=0.001 \\
\text { - Mucosal healing: VDL } 40.9 \% \text { vs. } 24.8 \% \text {, } \\
p=0.001 \\
\text { - Maintenance phase at W52: } \\
\text { - Clinical remission: VDLQ4 } 44.8 \% \text { vs. } \\
\text { VDLQ8 } 41.8 \% \text { vs. PBO } 15.9 \% \text { (all } p< \\
0.001 \text { vs. PBO) }\end{array}$ \\
\hline
\end{tabular}


TABLE 3 | Continued

Class of drug Agent

Trial

yms

RCT

Sample

Patient population

Treatment vs. comparison

Outcomes

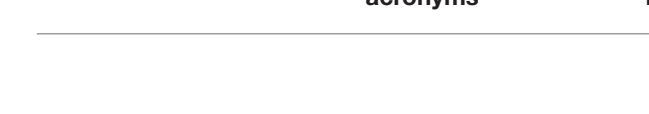

VDL vs. ADA VARSITY (185)

JAKi

TOF

OCTAVE
Induction-1 (186)

OCTAVE Induction-2

(186)

\section{OCTAVE}

-Sustain (186)

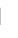

769

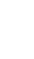

- Active UC despite corticosteroid, or immunomodulators (Non TNFi failure)

- 21\% Prior TNFi exposure

- $26 \%$ concomitant

immunomodulators

Active UC despite

immunomodulators/ TNF

74\% TNFi failure

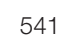

- Active UC despite

immunomodulators/ TNFi

- 70\% TNFi failure

593

- Patients who has a clinical

response in OCTAVE 1 and 2

- $T$. $10 \mathrm{mg} \mathrm{BD}, \mathrm{PO}$

- Vs. PBO

and 46 , IV

- ADA $40 \mathrm{mg} 160 \mathrm{mg}$ at WO, $80 \mathrm{mg}$ at

W2, then $40 \mathrm{mg}$ Q2W till W50, SC

- TOF $10 \mathrm{mg}$ BD, PO

- Vs. PBO

TOF $10 \mathrm{mg} \mathrm{BD,} \mathrm{PO}$

- Vs. PBO

No details yet

NCT02819635, NCT03653026, NCT03006068
- Mucosal healing: VDLQ4 56\% vs. VDLQ8 51.6\% vs. PBO 19.8\% (all $p<$ 0.001 vs. PBO)

- Clinical response at W52

VDL $31.3 \%$ vs. ADA 22.5\%, $p=0.006$ - Endoscopic improvement at W52:

VDL39.7\% vs. ADA 27.7\%; $P<0.001$.

Corticosteroid-free remission at W52:

VDL $12.6 \%$ vs. ADA $21.8 \%$, NS

- Clinical remission at W8:

TOF $18.5 \%$ vs. PBO $8.2 \%, p=0.007$

- Mucosal healing at W8:

TOF $31.3 \%$ vs. $15.6 \%, p<0.001$

- Clinical remission at W8:

TOF $16.6 \%$ vs. PBO 3.6\%, $p<0.001$

- Mucosal healing at W8: TOF $28.4 \%$ vs.

$11.6 \%, p<0.001$

- Clinical remission at W52:

TOF10 $40.6 \%$ vs. TOF5 $34.3 \%$ vs. PBO

$11.1 \%$, (all $p<0.001$ vs. PBO)

- Mucosal healing at W52:

TOF10 $45.7 \%$ vs. TOF5 $37.4 \%$ vs. PBO

$13.1 \%$, (all $p<0.001$ vs. PBO)

- Preliminary: met primary endpoints of clinical response, remission, endoscopic improvement, and response - No detail yet

ADA, adalimumab; $A S$, ankylosing spondylitis; axSpA, axial spondyloarthritis; AZA, azathioprine; BD, twice daily; Cl, confidence interval; CZP, certolizumab; GOL, golimumab; Gp, group; HR, hazard ratio: IFX, infliximab; IR, incidence rate ratio; IV, intravenous; JAKi, Janus kinase inhibitor; M, month; PO, per oral; Q, every; NS, not statistically significant: PBO, placebo; SC, subcutaneous; SEC, secukinumab; TNFi, tumor necrosis factor inhibitor: TOF, tofacitinib; UPA, upadacitinib; VDL, vedolizumab; Vs., versus; $W$, week; Yr, year. 
TABLE 4 | Evidence from clinical trials for class of therapeutic options for uveitis.

\begin{tabular}{|c|c|c|c|c|c|c|c|}
\hline $\begin{array}{l}\text { Class of } \\
\text { drug }\end{array}$ & Agent & $\begin{array}{l}\text { Trial } \\
\text { acronyms }\end{array}$ & $\begin{array}{l}\text { Trial } \\
\text { Phase }\end{array}$ & $\begin{array}{l}\text { Sample } \\
\text { size }\end{array}$ & $\begin{array}{l}\text { Patient } \\
\text { population }\end{array}$ & $\begin{array}{l}\text { Treatment vs. } \\
\text { comparison }\end{array}$ & Outcomes \\
\hline \multirow[t]{4}{*}{ TNFi } & ADA & VISUAL-1 (190) & RCT, III & 217 & $\begin{array}{l}\text { Active non-infectious } \\
\text { intermediate } \\
\text { uveitis, posterior uveitis, or } \\
\text { panuveitis despite } \\
\text { corticosteroid }\end{array}$ & $\begin{array}{l}\text { - ADA loading } 80 \mathrm{mg} \text {, then } 40 \mathrm{mg} \text { Q2W, } \\
\text { SC } \\
\text { - Vs. PBO }\end{array}$ & $\begin{array}{l}\text { - FU: till } 80 \mathrm{w} \text { or pre-specified events of } \\
\text { treatment failure is reached. } \\
\text { - Longer median time to treatment failure, } \\
\text { ADA vs PBO ( } 24 \mathrm{w} \text { vs. } 13 \mathrm{w} \text { ) } \\
\text { - ADA less likely than PBO group to have } \\
\text { treatment failure (HR } 0.50 ; 95 \% \mathrm{Cl} \text { : } \\
\text { 0.36-0.70; } P<0.001) \text {. }\end{array}$ \\
\hline & ADA & VISUAL-2 (191) & RCT, III & 229 & $\begin{array}{l}\text { Inactive, non-infectious } \\
\text { intermediate, posterior, or } \\
\text { panuveitis requiring } \\
\text { prednisolone for } \\
\text { maintenance }\end{array}$ & $\begin{array}{l}\text { - ADA loading } 80 \mathrm{mg} \text {, then } 40 \mathrm{mg} \text { Q2W, } \\
\text { SC } \\
\text { - Vs. PBO }\end{array}$ & $\begin{array}{l}\text { - FU: till } 80 \mathrm{w} \text { or at treatment failure event } \\
\text { - Long time to treatment failure, ADA vs } \\
\text { PBO (10.2m vs. } 4.8 \mathrm{~m}) \\
\text { - ADA less likely than PBO group to have } \\
\text { treatment failure (HR 0.57; } 95 \% \mathrm{Cl} \text { : } \\
\text { 0.39-.84; } P=0.004) .\end{array}$ \\
\hline & GOL & GO-EASY (192) & $\begin{array}{l}\text { Open label, } \\
\text { non-randomized }\end{array}$ & 93 & $\begin{array}{l}\text { - AS patients ( } 55 \% \\
\text { TNFi-naive, } 27 \% \text { history of } \\
\text { uveitis) }\end{array}$ & $\begin{array}{l}\text { All: GOL } 50 \text { mg monthly } \\
\text { - VS. historical control (flare rates in } \\
\text { previous yr) }\end{array}$ & $\begin{array}{l}\text { - Lower risk of uveitis flare in GOL vs. } \\
\text { historical rates }(2.2 \text { vs. } 11.1 \text { per } 100 \\
\text { patient-years, rate-ratio } 0.20,95 \% \mathrm{Cl} \\
0.04-0.91) \text {. }\end{array}$ \\
\hline & CZP & $\begin{array}{l}\text { (Abstract only) } \\
\text { (193) }\end{array}$ & $\begin{array}{l}\text { Open label, non- } \\
\text { randomized, } \\
\text { IV }\end{array}$ & $\begin{array}{l}115 \text { enrolled } \\
\text { (85 in interim } \\
\text { analysis) }\end{array}$ & $\begin{array}{l}\text { Active axSpA, HLAB27 } \\
\text { positive, having history of } \\
\text { recurrent uveitis }\end{array}$ & $\begin{array}{l}\text { - All: } 400 \mathrm{mg} \text { at W0, 2, 4, then } 200 \mathrm{mg} \\
\text { Q2W till W96 } \\
\text { - Vs. historical control }\end{array}$ & $\begin{array}{l}\text { - Interim analysis of } 85 \text { patients completed } \\
\text { W48 } \\
\text { - Few flares CZP vs. historical rates } \\
\text { (Poisson-adjusted IR: } 0.2 \text { vs } 1.5, p< \\
\text { 0.001). }\end{array}$ \\
\hline \multirow[t]{2}{*}{ IL-17i } & SEC & $\begin{array}{l}3 \text { RCTS: } \\
\text { SHIELD, } \\
\text { INSURE, } \\
\text { ENDURE (194) }\end{array}$ & RCT, III & 274 & $\begin{array}{l}\text { Behçet's uveitis }=118 \\
\text { (SHIELD) } \\
\text { Active non-infectious active } \\
\text { uveitis }=31 \text { (INSURE) } \\
\text { Inactive non-infectious } \\
\text { uveitis }=125 \text { (ENDURE) }\end{array}$ & $\begin{array}{l}\text { - Varies dosing: } \\
\text { - SEC loading ( } 150 \mathrm{mg} \text { or } 300 \mathrm{mg} \text { ), then } \\
\text { Q2W-Q4W } \\
\text { - Vs. PBO }\end{array}$ & $\begin{array}{l}\text { - SHIELD: completed, primary endpoint } \\
\text { not met } \\
\text { - INSURE: terminated early } \\
\text { - ENDURE: completed, planned analysis } \\
\text { dropped } \\
\text { - No statistically significant differences in } \\
\text { uveitis flares, SEC vs. PBO in all } 3 \text { RCTs }\end{array}$ \\
\hline & SEC & (195) & $\|$ & 37 & $\begin{array}{l}\text { Active non-infectious } \\
\text { intermediate uveitis, } \\
\text { posterior uveitis, or } \\
\text { panuveitis, requiring } \\
\text { corticosteroid sparing } \\
\text { therapy }\end{array}$ & $\begin{array}{l}\text { - SEC } 30 \mathrm{mg} / \mathrm{kg} \text { Q4W, IV for } 2 \text { doses, } \\
\text { (Group 1) } \\
\text { - Vs. SEC } 10 \mathrm{mg} / \mathrm{kg} \text { Q2W, IV for } 4 \text { doses, } \\
\text { (Gp 2) } \\
\text { - Vs. SEC } 300 \mathrm{mg} \text { Q2W, SC for } 4 \text { doses, } \\
\text { (Gp 3) }\end{array}$ & $\begin{array}{l}\text { - Higher response rate in higher dose } \\
\text { compared to lower dose regimen on day } \\
57 . \\
\text { - Responder rates (Gp 1: } 72.7 \% \text { vs. } \\
\text { Gp2: } 61.5 \% \text { vs. Gp3: } 33.3 \% \text {, statistically } \\
\text { significant Gp } 1 \text { vs. Gp3) } \\
\text { - Remission rates (Gp1: } 27.3 \% \text { and Gp2: } \\
\text { 38.5\% vs. Gp3: } 16.7 \%, N S \text { ) }\end{array}$ \\
\hline \multirow[t]{2}{*}{ IL-12/23i } & UST & STAR (196) & $\|$ & 8 enrolled & $\begin{array}{l}\text { Active sight-threatening } \\
\text { active intermediate uveitis, } \\
\text { posterior uveitis, or } \\
\text { panuveitis }\end{array}$ & $\begin{array}{l}-90 \mathrm{mg}, \mathrm{SC} \text { at W0,4 and } 8 \text { vs } \\
260-520 \mathrm{mg} \text { (weight-based dose), IV at } \\
\text { W0 then } 90 \mathrm{mg}, \mathrm{SC} \text { at W8 }\end{array}$ & $\begin{array}{l}\text { - Completed, awaiting analysis and } \\
\text { publication of results }\end{array}$ \\
\hline & UST & $\begin{array}{l}\text { STELABEC-2 } \\
\text { (197) }\end{array}$ & $\|$ & 16 & $\begin{array}{l}\text { Active posterior uveitis } \\
\text { and/or panuveitis and/or } \\
\text { retinal vasculitis in patients } \\
\text { with Behçet's disease }\end{array}$ & $\begin{array}{l}\text { - } 90 \mathrm{mg}, \mathrm{SC} \text { at WO, W4, and W16. } \\
\text { Patients with response will receive } \\
90 \mathrm{mg}, \mathrm{SC} \text { at W28 and W40 }\end{array}$ & - Ongoing \\
\hline
\end{tabular}

ADA, adalimumab; AS, ankylosing spondylitis; axSpA, axial spondyloarthritis; Cl, confidence interval; CZP, certolizumab; GOL, golimumab; Gp, group; HR, hazard ratio; IR, incidence rate ratio; IV, intravenous; $M$, month; $Q$, every; NS, not statistically significant; PBO, placebo; SC, subcutaneous; SEC, secukinumab; TNFi, tumor necrosis factor inhibitor; UST, ustekinumab; Vs., versus; W, week; Yr, year. 
therapeutics to create a patient-centric, individualized care plan for the heterogeneous manifestations. Often, the therapeutics will need to cover multiple domains, but the predominant domain should drive the therapeutic option of choice in the shared decision-making process.

For severe IBD in the setting of PsA with peripheral manifestations, traditional immunomodulators can be considered for maintenance. TNFi (monoclonal antibodies) is a better option for patients with axial arthropathy. UST is effective for IBD but is less effective on peripheral arthritis as compared to TNFi or IL-17i, and ineffective for axial arthropathy. While IL-17i is an effective treatment for both peripheral and axial arthropathy, and probably does not increase the risk of IBD, it is not recommended for patients with underlying active IBD, due to its possibility of exacerbating pre-existing disease. IL-23i may be promising for IBD but its use requires caution in patients with predominant axial arthropathy. JAKi is effective for UC, peripheral and axial arthropathy, but may exacerbate CD. VDZ is effective for both CD and UC but has no indication for all other manifestations in PsA. With these considerations, TNFi (monoclonal antibodies) with or without concomitant immunosuppressants would be the best option for PsA patients with IBD. IBD is a chronic relapsing condition, and often requires higher doses of TNFi for induction than arthritis alone. Collaboration between rheumatologist and gastroenterologist is invaluable to ensure the optimal choice of treatment regimen.

Uveitis can be serious and sight threatening. Patients with symptoms of possible uveitis should have access to ophthalmology care promptly and given appropriate treatment for uveitis. Uveitis can arise even when arthritis is under control; it may manifest either suddenly or insidiously. It is important that patients are educated to be aware of the symptoms of uveitis and seek appropriate care when the needs arise. Care models like enquiry hotline, early referral or walk-in ophthalmology clinics are examples that may facilitate early diagnosis. For subsequent management, collaboration between rheumatologist and ophthalmologist is essential to ensure regular assessment of response to therapy and to modify management accordingly.

\section{REFERENCES}

1. Ritchlin CT, Colbert RA, Gladman DD. Psoriatic Arthritis. New England Journal of Medicine. (2017) 376:957-70. doi: 10.1056/NEJMra1505 557

2. Orbai AM, de Wit M, Mease P, Shea JA, Gossec L, Leung YY, et al. International patient and physician consensus on a psoriatic arthritis core outcome set for clinical trials. Ann Rheum Dis. (2017) 76:673-80. doi: 10.1136/annrheumdis-2016-210242

3. Husni ME, Merola JF, Davin S. The psychosocial burden of psoriatic arthritis. Semin Arthritis Rheum. (2017) 47:351-60. doi: 10.1016/j.semarthrit.2017.05.010

4. So H, Tam LS. Cardiovascular disease and depression in psoriatic arthritis: multidimensional comorbidities requiring multidisciplinary management. Best Pract Res Clin Rheumatol. (2021) 101689. doi: 10.1016/j.berh.2021.101689

5. Kovitwanichkanont T, Chong AH, Foley P. Beyond skin deep: addressing comorbidities in psoriasis. Med J Aust. (2020) 212:528-34. doi: $10.5694 / \mathrm{mja} 2.50591$
If uveitis fails to respond to topical corticosteroids, or fails to be weaned, or is severe at the onset, an escalation to either conventional immunomodulators or biological agents should be considered. The use of systemic corticosteroid is best avoided, given the risk of severe PsO flare upon its withdrawal. For patients with peripheral musculoskeletal manifestations (peripheral arthritis, enthesitis and dactylitis), MTX, SSZ or leflunomide (LEF) can be tried for maintenance, but an early escalation to TNFi (monoclonal antibodies) should be considered if these options fail. Traditional immunomodulators are not effective for axial arthropathy, thus for patients with active axial arthropathy TNFi (monoclonal antibodies) would be a good choice. Some patients may require higher or more frequent doses of TNFi especially for severe uveitis, highlighting again the importance of collaboration between rheumatologist and ophthalmologist for drug titration. IL-17i is an effective treatment for axial arthropathy, but SEC may not be effective for AAU at standard dose, and more data is still needed to inform the use of other IL-17i.

All in all, detailed considerations of all domains and extraarticular manifestations are necessary to formulate the best therapeutic option. Multi-disciplinary collaborative care models are advocated for optimal care for patients with PsA, and especially so for those who present with co-morbidities.

\section{AUTHOR CONTRIBUTIONS}

$\mathrm{AlC}$ and $\mathrm{YL}$ conceptualized the project. AlC, TC, and YL collected relevant data for review and drafted the manuscript. AnC, WC, GA, and YL critically appraised the content of manuscript. All authors approved the final version of manuscript.

\section{FUNDING}

YL was supported by the National Medical Research Council, Singapore (NMRC/CSA-Inv/0022/2017). The funding sources had no role in the views expressed in this review.

6. Noviani M, Feletar M, Nash P, Leung YY. Choosing the right treatment for patients with psoriatic arthritis. Ther Adv Musculoskelet Dis. (2020). 12:1759720x20962623. doi: 10.1177/1759720X20962623

7. Yu YR, Rodriguez JR. Clinical presentation of Crohn's, ulcerative colitis, and indeterminate colitis: Symptoms, extraintestinal manifestations, disease phenotypes. Semin Pediatr Surg. (2017) 26:349-55. doi: 10.1053/j.sempedsurg.2017.10.003

8. Langholz E. Current trends in inflammatory bowel disease: the natural history. Therap Adv Gastroenterol. (2010) 3:77-86. doi: 10.1177/1756283X10361304

9. Arvikar SL, Fisher MC. Inflammatory bowel disease associated arthropathy. Curr Rev Musculoskelet Med. (2011) 4:123-31. doi: 10.1007/s12178-011-9085-8

10. Larsen S, Bendtzen K, Nielsen OH. Extraintestinal manifestations of inflammatory bowel disease: epidemiology, diagnosis, and management. Ann Med. (2010) 42:97-114. doi: 10.3109/07853890903559724

11. Rodríguez-Reyna TS, Martínez-Reyes C, Yamamoto-Furusho JK. Rheumatic manifestations of inflammatory bowel disease. World J Gastroenterol. (2009) 15:5517-24. doi: 10.3748/wjg.15.5517 
12. Atzeni F, Defendenti C, Ditto MC, Batticciotto A, Ventura D, Antivalle M, et al. Rheumatic manifestations in inflammatory bowel disease. Autoimmun Rev. (2014) 13:20-3. doi: 10.1016/j.autrev.2013.06.006

13. Orlando A, Renna S, Perricone G, Cottone M. Gastrointestinal lesions associated with spondyloarthropathies. World J Gastroenterol. (2009) 15:2443-8. doi: 10.3748/wjg.15.2443

14. Li, W-Q, Han J-L, Chan AT, Qureshi AA. Psoriasis, psoriatic arthritis and increased risk of incident Crohn's disease in US women. Ann Rheumatic Dis. (2013). 72:1200-5. doi: 10.1136/annrheumdis-2012-202143

15. Schreiber S, Colombel J-F, Feagan BG, Reich K, Deodhar AA, McInnes IB, et al. Incidence rates of inflammatory bowel disease in patients with psoriasis, psoriatic arthritis and ankylosing spondylitis treated with secukinumab: a retrospective analysis of pooled data from 21 clinical trials. Ann Rheum Dis. (2019) 78:473-9. doi: 10.1136/annrheumdis-2018-214273

16. Zohar A, Cohen AD, Bitterman H, Feldhamer I, Greenberg-Dotan S, Lavi I, et al. Gastrointestinal comorbidities in patients with psoriatic arthritis. Clin Rheumatol. (2016) 35:2679-84. doi: 10.1007/s10067-016-3374-y

17. Makredes M, Robinson D, Bala M, Kimball AB. The burden of autoimmune disease: a comparison of prevalence ratios in patients with psoriatic arthritis and psoriasis. J Am Acad Dermatol. (2009) 61:405-10. doi: 10.1016/j.jaad.2009.02.015

18. Eppinga $H$, Poortinga S, Thio HB, Nijsten TEC, Nuij V, van der Woude CJ, et al. Prevalence and phenotype of concurrent psoriasis and inflammatory bowel disease. Inflamm Bowel Dis. (2017) 23:1783-9. doi: 10.1097/MIB.0000000000001169

19. Kopylov U, Starr M, Watts C, Dionne S, Girardin M, Seidman EG. Detection of crohn disease in patients with spondyloarthropathy: the SpACE capsule study. J Rheumatol. (2018) 45:498-505. doi: 10.3899/jrheum.161216

20. Mielants H, Veys EM, Cuvelier C, de Vos M. Ileocolonoscopic findings in seronegative spondylarthropathies. Br J Rheumatol. (1988) 27 Suppl 2:95105. doi: 10.1093/rheumatology/XXVII.suppl_2.95

21. Lee YH Ji JD, Kim JS, Bak YT, Lee CH, Kim CH, et al. Ileocolonoscopic and histologic studies of Korean patients with ankylosing spondylitis. Scand J Rheumatol. (1997) 26:473-6. doi: 10.3109/03009749709065722

22. Leirisalo-Repo M, Turunen U, Stenman S, Helenius P, Seppälä K. High frequency of silent inflammatory bowel disease in spondylarthropathy. Arthritis Rheum. (1994) 37:23-31. doi: 10.1002/art.1780370105

23. Van Praet L, Van den Bosch FE, Jacques P, Carron P, Jans L, Colman $\mathrm{R}$, et al. Microscopic gut inflammation in axial spondyloarthritis: a multiparametric predictive model. Ann Rheum Dis. (2013) 72:414-7. doi: 10.1136/annrheumdis-2012-202135

24. Van Praet L, Jans L, Carron P, Jacques P, Glorieus E, Colman R, et al. Degree of bone marrow oedema in sacroiliac joints of patients with axial spondyloarthritis is linked to gut inflammation and male sex: results from the GIANT cohort. Ann Rheum Dis. (2014) 73:1186-9. doi: 10.1136/annrheumdis-2013-203854

25. De Vos M, Mielants H, Cuvelier C, Elewaut A, Veys E. Long-term evolution of gut inflammation in patients with spondyloarthropathy. Gastroenterology. (1996) 110:1696-703. doi: 10.1053/gast.1996.v110.pm8964393

26. Stolwijk C, van Tubergen A, Castillo-Ortiz JD, Boonen A. Prevalence of extra-articular manifestations in patients with ankylosing spondylitis: a systematic review and meta-analysis. Ann Rheum Dis. (2015) 74:65-73. doi: 10.1136/annrheumdis-2013-203582

27. Stolwijk C, Essers I, van Tubergen A, Boonen A, Bazelier MT, De Bruin $\mathrm{ML}$, et al. The epidemiology of extra-articular manifestations in ankylosing spondylitis: a population-based matched cohort study. Ann Rheum Dis. (2015) 74:1373-8. doi: 10.1136/annrheumdis-2014-205253

28. de Winter JJ, van Mens LJ, van der Heijde D, Landewé R, Baeten DL. Prevalence of peripheral and extra-articular disease in ankylosing spondylitis versus non-radiographic axial spondyloarthritis: a meta-analysis. Arthritis Res Ther. (2016) 18:196. doi: 10.1186/s13075-016-1093-z

29. Sanchez-Bilbao L, Martinez-Lopez D, Palmou-Fontana N, Armesto S, González-Gay MA, Blanco R. AB0829 inflammatoy bowel disease in psoriatic arthritis. Study of 306 patients from a single university center, prevalence clinical features and relationship to biologic therapy. Ann Rheum Dis. (2020) 79:1719-1719. doi: 10.1136/annrheumdis-2020-eular.4806

30. Jadon DR, Sengupta R, Nightingale A, Lindsay M, Korendowych E, Robinson $\mathrm{G}$, et al. Axial Disease in Psoriatic Arthritis study: defining the clinical and radiographic phenotype of psoriatic spondyloarthritis. Ann Rheum Dis. (2017) 76:701-7. doi: 10.1136/annrheumdis-2016-209853

31. Lee RW, Nicholson LB, Sen HN, Chan C-C, Wei L, Nussenblatt RB, et al. Autoimmune and autoinflammatory mechanisms in uveitis. Semin Immunopathol. (2014) 36:581-94. doi: 10.1007/s00281-014-0433-9

32. Li, C-R, Chen L, Wang L-F, Yan B, Liang Y-L, Luo J. Association between uveitis and psoriatic disease: a systematic review and Meta-analysis based on the evidence from cohort studies. Int J Ophthalmol. (2020). 13:650-9. doi: 10.18240/ijo.2020.04.19

33. Ruggiero A, Fabbrocini G, Cacciapuoti S, Cinelli E, Gallo L, Megna M. Ocular manifestations in psoriasis screening (OcMaPS) questionnaire: a useful tool to reveal misdiagnosed ocular involvement in psoriasis. J Clin Med. (2021). 10:1031. doi: 10.3390/jcm10051031

34. Abbouda A, Abicca I, Fabiani C, Scappatura N, Peña-García P, Scrivo R, et al. Psoriasis and psoriatic arthritis-related uveitis: different ophthalmological manifestations and ocular inflammation features. Semin Ophthalmol. (2017) 32:715-20. doi: 10.3109/08820538.2016.1170161

35. Castellino N, Longo A, Fallico M, Russo A, Bonfiglio V, Cennamo G, et al. Retinal vascular assessment in psoriasis: a multicenter study. Front Neurosci. (2021) 15:629401. doi: 10.3389/fnins.2021.629401

36. Zeboulon N, Dougados M, Gossec L. Prevalence and characteristics of uveitis in the spondyloarthropathies: a systematic literature review. Ann Rheum Dis. (2008) 67:955-9. doi: 10.1136/ard.2007.075754

37. Niccoli L, Nannini C, Cassarà E, Kaloudi O, Susini M, Lenzetti I, et al. Frequency of iridocyclitis in patients with early psoriatic arthritis: a prospective, follow up study. Int J Rheum Dis. (2012) 15:414-8. doi: 10.1111/j.1756-185X.2012.01736.X

38. Egeberg A, Khalid U, Gislason GH, Mallbris L, Skov L, Hansen PR. Association of psoriatic disease with uveitis: a danish nationwide cohort study. JAMA Dermatol. (2015) 151:1200-5 doi: 10.1001/jamadermatol.2015.1986

39. Chi CC, Tung TH, Wang J, Lin YS, Chen YF, Hsu TK, et al. Risk of uveitis among people with psoriasis: a nationwide cohort study. JAMA Ophthalmol. (2017) 135:415-22. doi: 10.1001/jamaophthalmol.2017.0569

40. Khan MA, Haroon M, Rosenbaum JT. Acute anterior uveitis and spondyloarthritis: more than meets the eye. Curr Rheumatol Rep. (2015) 17:59. doi: 10.1007/s11926-015-0536-x

41. Paiva ES, Macaluso DC, Edwards A, Rosenbaum JT. Characterisation of uveitis in patients with psoriatic arthritis. Ann Rheum Dis. (2000) 59:67-70. doi: 10.1136/ard.59.1.67

42. Peluso R, Iervolino S, Vitiello M, Bruner V, Lupoli G, Di Minno MN. Extraarticular manifestations in psoriatic arthritis patients. Clin Rheumatol. (2015) 34:745-53. doi: 10.1007/s10067-014-2652-9

43. Queiro R, Torre JC, Belzunegui J, González C, De Dios JR, Unanue F, et al. Clinical features and predictive factors in psoriatic arthritis-related uveitis. Semin Arthritis Rheum. (2002) 31:264-70. doi: 10.1053/sarh.2002.28798

44. Burden-Teh E, Murphy R. Psoriasis and uveitis-should we be asking about eye symptoms? Br J Dermatol. (2014) 170:756-7. doi: 10.1111/bjd.12731

45. O'Rielly DD, Rahman P. Clinical and molecular significance of genetic loci associated with psoriatic arthritis. Best Pract Res Clin Rheumatol. (2021) 35:101691. doi: 10.1016/j.berh.2021.101691

46. Haroon M, Winchester R, Giles JT, Heffernan E, FitzGerald O. Certain class I HLA alleles and haplotypes implicated in susceptibility play a role in determining specific features of the psoriatic arthritis phenotype. Ann Rheum Dis. (2016) 75:155-62. doi: 10.1136/annrheumdis-2014-205461

47. Winchester R, Minevich G, Steshenko V, Kirby B, Kane D, Greenberg $\mathrm{DA}$, et al. associations reveal genetic heterogeneity in psoriatic arthritis and in the psoriasis phenotype. Arthritis Rheum. (2012) 64:1134-44. doi: 10.1002/art.33415

48. Murray C, Mann DL, Gerber LN, Barth W, Perlmann S, Decker JL, et al. Histocompatibility alloantigens in psoriasis and psoriatic arthritis. Evidence for the influence of multiple genes in the major histocompatibility complex. J Clin Invest. (1980) 66:670-5. doi: 10.1172/JCI10 9903

49. Eastmond CJ. Psoriatic arthritis. Genetics and HLA antigens Baillieres. Clin Rheumatol. (1994) 8:263-76. doi: 10.1016/S0950-3579(94)80018-9

50. Gladman DD, Farewell VT, Kopciuk KA, Cook RJ, HLA. markers and progression in psoriatic arthritis. J Rheumatol. (1998) 25:730-3. 
51. Espinoza LR, Vasey FB, Oh JH, Wilkinson R, Osterland CK. Association between HLA-BW38 and peripheral psoriatic arthritis. Arthritis Rheum. (1978) 21:72-5. doi: 10.1002/art.1780210112

52. Bowes J, Budu-Aggrey A, Huffmeier U, Uebe S, Steel K, Hebert HL, et al. Dense genotyping of immune-related susceptibility loci reveals new insights into the genetics of psoriatic arthritis. Nat Commun. (2015) 6:6046. doi: $10.1038 /$ ncomms 8741

53. Hüffmeier U, Uebe S, Ekici AB, Bowes J, Giardina E, Korendowych E, et al. Common variants at TRAF3IP2 are associated with susceptibility to psoriatic arthritis and psoriasis. Nat Genet. (2010) 42:996-9. doi: 10.1038/ng.688

54. Stuart PE, Nair RP, Tsoi LC, Tejasvi T, Das S, Kang HM, et al. Genomewide association analysis of psoriatic arthritis and cutaneous psoriasis reveals differences in their genetic architecture. Am J Hum Genet. (2015) 97:816-36. doi: 10.1016/j.ajhg.2015.10.019

55. Doyle MS, Collins ES, FitzGerald OM, Pennington SR. New insight into the functions of the interleukin-17 receptor adaptor protein Act1 in psoriatic arthritis. Arthritis Res Ther. (2012) 14:226. doi: 10.1186/ar4071

56. Vecellio M, Hake VX, Davidson C, Carena MC, Wordsworth BP, Selmi C. The IL-17/IL-23 axis and its genetic contribution to psoriatic arthritis. Front Immunol. (2020) 11:596086. doi: 10.3389/fimmu.2020.596086

57. Jacques P, Lambrecht S, Verheugen E, Pauwels E, Kollias G, Armaka M, et al. Proof of concept: enthesitis and new bone formation in spondyloarthritis are driven by mechanical strain and stromal cells. Ann Rheum Dis. (2014) 73:437-45. doi: 10.1136/annrheumdis-2013-203643

58. Schett G, Lories RJ, D'Agostino MA, Elewaut D, Kirkham B, Soriano ER, et al. Enthesitis: from pathophysiology to treatment. Nat Rev Rheumatol. (2017) 13:731-41. doi: 10.1038/nrrheum.2017.188

59. Wilson NJ, Boniface K, Chan JR, McKenzie BS, Blumenschein WM, Mattson JD, et al. Development, cytokine profile and function of human interleukin 17-producing helper T cells. Nat Immunol. (2007) 8:950-7. doi: 10.1038/ni1497

60. Paulissen SM, van Hamburg JP, Davelaar N, Asmawidjaja PS, Hazes JM, Lubberts E. Synovial fibroblasts directly induce Th17 pathogenicity via the cyclooxygenase/prostaglandin E2 pathway, independent of IL-23. J Immunol. (2013) 191:1364-72. doi: 10.4049/jimmunol.1300274

61. McGonagle D, McDermott MF, A. proposed classification of the immunological diseases. PLoS Med. (2006) 3:e297. doi: 10.1371/journal.pmed.0030297

62. Jongbloed SL, Lebre MC, Fraser AR, Gracie JA, Sturrock RD, Tak PP, et al. Enumeration and phenotypical analysis of distinct dendritic cell subsets in psoriatic arthritis and rheumatoid arthritis. Arthritis Res Ther. (2006) 8:R15. doi: $10.1186 /$ ar1864

63. Candia L, Marquez J, Hernandez C, Zea AH, Espinoza LR. Toll-like receptor2 expression is upregulated in antigen-presenting cells from patients with psoriatic arthritis: a pathogenic role for innate immunity? J Rheumatol. (2007) 34:374-9.

64. Mease P. TNFalpha therapy in psoriatic arthritis and psoriasis. Ann Rheum Dis. (2004) 63:755-8. doi: 10.1136/ard.2004.020719

65. Leijten EF, van Kempen TS, Boes M. Michels-van Amelsfort JM, Hijnen D, Hartgring SA, et al. Brief report: enrichment of activated group 3 innate lymphoid cells in psoriatic arthritis synovial fluid. Arthritis Rheumatol. (2015) 67:2673-8. doi: 10.1002/art.39261

66. Soare A, Weber S, Maul L, Rauber S, Gheorghiu AM, Luber M, et al. Cutting edge: homeostasis of innate lymphoid cells is imbalanced in psoriatic arthritis. J Immunol. (2018) 200:1249-54. doi: 10.4049/jimmunol.1700596

67. Teunissen MBM, Yeremenko NG, Baeten DLP, Chielie S, Spuls PI, de Rie MA, et al. The IL-17A-producing CD8+ T-cell population in psoriatic lesional skin comprises mucosa-associated invariant $\mathrm{T}$ cells and conventional T cells. J Invest Dermatol. (2014) 134:2898-907. doi: 10.1038/jid.2014.261

68. Raychaudhuri SK, Abria C, Mitra A, Raychaudhuri SP. Functional significance of MAIT cells in psoriatic arthritis. Cytokine. (2020) 125:154855. doi: 10.1016/j.cyto.2019.154855

69. Guggino G, Ciccia F, Di Liberto D, Lo Pizzo M, Ruscitti P, Cipriani P, et al. Interleukin (IL)-9/IL-9R axis drives $\gamma \delta \mathrm{T}$ cells activation in psoriatic arthritis patients. Clin Exp Immunol. (2016) 186:277-83. doi: 10.1111/cei.12853

70. Miossec P. Local and systemic effects of IL-17 in joint inflammation: a historical perspective from discovery to targeting. Cell Mol Immunol. (2021) 18:860-5. doi: 10.1038/s41423-021-00644-5
71. Chizzolini C, Chicheportiche R, Alvarez M, de Rham C, RouxLombard P, Ferrari-Lacraz S, et al. Prostaglandin E2 synergistically with interleukin-23 favors human Th17 expansion. Blood. (2008) 112:3696-703. doi: 10.1182/blood-2008-05-155408

72. Menon B, Gullick NJ, Walter GJ, Rajasekhar M, Garrood T, Evans HG, et al. Interleukin-17+CD8+ T cells are enriched in the joints of patients with psoriatic arthritis and correlate with disease activity and joint damage progression. Arthritis Rheumatol. (2014) 66:1272-81. doi: 10.1002/art.38376

73. Steel KJA, Srenathan U, Ridley M, Durham LE, Wu S-Y, Ryan SE, et al. Polyfunctional Proinflammatory, Tissue-Resident Memory Phenotype and Function of Synovial Interleukin-17A+CD8+ T Cells in Psoriatic Arthritis. Arthritis Rheumatol. (2020) 72:435-47. doi: 10.1002/art.41156

74. Raychaudhuri SK, Saxena A, Raychaudhuri SP. Role of IL-17 in the pathogenesis of psoriatic arthritis and axial spondyloarthritis. Clin Rheumatol. (2015) 34:1019-23. doi: 10.1007/s10067-015-2961-7

75. Benham H, Norris P, Goodall J, Wechalekar MD, FitzGerald O, Szentpetery A, et al. Th17 and Th22 cells in psoriatic arthritis and psoriasis. Arthritis Res Ther. (2013) 15:R136-R136. doi: 10.1186/ar4317

76. Salvarani C, Fries W. Clinical features and epidemiology of spondyloarthritides associated with inflammatory bowel disease. World $J$ Gastroenterol. (2009) 15:2449-55. doi: 10.3748/wjg.15.2449

77. Sheth T, Pitchumoni CS, Das KM. Management of musculoskeletal manifestations in inflammatory bowel disease. Gastroenterol Res Pract. (2015) 2015:387891. doi: 10.1155/2015/387891

78. Goyette P, Boucher G, Mallon D, Ellinghaus E, Jostins L, Huang H, et al. High-density mapping of the MHC identifies a shared role for HLADRB1*01:03 in inflammatory bowel diseases and heterozygous advantage in ulcerative colitis. Nat Genet. (2015) 47:172-9. doi: 10.1038/ng.3176

79. Orchard TR, Thiyagaraja S, Welsh KI, Wordsworth BP, Hill Gaston JS, Jewell DP. Clinical phenotype is related to HLA genotype in the peripheral arthropathies of inflammatory bowel disease. Gastroenterology. (2000) 118:274-8. doi: 10.1016/S0016-5085(00)70209-5

80. Reveille JD, Zhou X, Lee M, Weisman MH Yi L, Gensler LS, et al. HLA class I and II alleles in susceptibility to ankylosing spondylitis. Ann Rheum Dis. (2019) 78:66-73. doi: 10.1136/annrheumdis-2018-213779

81. Bouma G, Strober W. The immunological and genetic basis of inflammatory bowel disease. Nat Rev Immunol. (2003) 3:521-33. doi: 10.1038/nri1132

82. Brakenhoff LK, van der Heijde DM, Hommes DW, Huizinga TW, Fidder HH. The joint-gut axis in inflammatory bowel diseases. J Crohns Colitis. (2010) 4:257-68. doi: 10.1016/j.crohns.2009.11.005

83. Peeters H, Vander Cruyssen B, Laukens D, Coucke P, Marichal D, Van Den Berghe M, et al. Radiological sacroiliitis, a hallmark of spondylitis, is linked with CARD15 gene polymorphisms in patients with Crohn's disease. Ann Rheum Dis. (2004) 63:1131-4. doi: 10.1136/ard.2004.021774

84. Ogura Y, Bonen DK, Inohara N, Nicolae DL, Chen FF, Ramos R, et al. A frameshift mutation in NOD2 associated with susceptibility to Crohn's disease. Nature. (2001) 411:603-6. doi: 10.1038/35079114

85. Liu T, Zhang L, Joo D, Sun S-C. NF- $\mathrm{B}$ signaling in inflammation. Signal Transduct Targeted Ther. (2017) 2:17023. doi: 10.1038/sigtrans.2017.23

86. Duerr RH, Taylor KD, Brant SR, Rioux JD, Silverberg MS, Daly MJ, et al. A genome-wide association study identifies IL23R as an inflammatory bowel disease gene. Science. (2006) 314:1461-3. doi: 10.1126/science.113 5245

87. Danoy P, Pryce K, Hadler J, Bradbury LA, Farrar C, Pointon J, et al. Association of variants at 1q32 and STAT3 with ankylosing spondylitis suggests genetic overlap with Crohn's disease. PLoS Genet. (2010) 6:e1001195-e1001195. doi: 10.1371/journal.pgen.1001195

88. Ott SJ, Musfeldt M, Wenderoth DF, Hampe J, Brant O, Fölsch UR, et al. Reduction in diversity of the colonic mucosa associated bacterial microflora in patients with active inflammatory bowel disease. Gut. (2004) 53:685-93. doi: 10.1136/gut.2003.025403

89. Martinez-Medina M, Aldeguer X, Gonzalez-Huix F, Acero D, GarciaGil LJ. Abnormal microbiota composition in the ileocolonic mucosa of Crohn's disease patients as revealed by polymerase chain reactiondenaturing gradient gel electrophoresis. Inflamm Bowel Dis. (2006) 12:113645. doi: 10.1097/01.mib.0000235828.09305.0c

90. Johansson ME, Phillipson M, Petersson J, Velcich A, Holm L, Hansson GC. The inner of the two Muc2 mucin-dependent mucus layers in colon 
is devoid of bacteria. Proc Natl Acad Sci U S A. (2008) 105:15064-9. doi: 10.1073/pnas. 0803124105

91. Gracey E, Vereecke L, McGovern D, Fröhling M, Schett G, Danese $\mathrm{S}$, et al. Revisiting the gut-joint axis: links between gut inflammation and spondyloarthritis. Nat Rev Rheumatol. (2020) 16:415-33. doi: 10.1038/s41584-020-0454-9

92. Jacques $\mathrm{P}$, Elewaut $\mathrm{D}$, Mielants $\mathrm{H}$. Interactions between gut inflammation and arthritis/spondylitis. Curr Opin Rheumatol. (2010) 22:368-74. doi: 10.1097/BOR.0b013e3283393807

93. Imdad A, Nicholson MR, Tanner-Smith EE, Zackular JP, GomezDuarte OG, Beaulieu DB, et al. Fecal transplantation for treatment of inflammatory bowel disease. Cochrane Database Syst Rev. (2018) 11:Cd012774. doi: 10.1002/14651858.CD012774.pub2

94. Zhao HL, Chen SZ, Xu HM, Zhou YL, He J, Huang HL, et al. Efficacy and safety of fecal microbiota transplantation for treating patients with ulcerative colitis: A systematic review and meta-analysis. J Dig Dis. (2020) 21:534-48. doi: 10.1111/1751-2980.12933

95. Moayyedi P, Surette MG, Kim PT, Libertucci J, Wolfe M, Onischi C, et al. Fecal microbiota transplantation induces remission in patients with active ulcerative colitis in a randomized controlled trial. Gastroenterology. (2015). 149:102-109e6. doi: 10.1053/j.gastro.2015.04.001

96. Kragsnaes MS, Kjeldsen J, Horn HC, Munk HL, Pedersen JK, Just SA, et al. Safety and efficacy of faecal microbiota transplantation for active peripheral psoriatic arthritis: an exploratory randomised placebo-controlled trial. Ann Rheum Dis. (2021). doi: 10.1136/annrheumdis-2021-220910

97. Serriari NE, Eoche M, Lamotte L, Lion J, Fumery M, Marcelo P, et al. Innate mucosal-associated invariant $\mathrm{T}$ (MAIT) cells are activated in inflammatory bowel diseases. Clin Exp Immunol. (2014) 176:266-74. doi: 10.1111/cei.12277

98. Deusch K, Lüling F, Reich K, Classen M, Wagner H, Pfeffer K, et al. major fraction of human intraepithelial lymphocytes simultaneously expresses the gamma/delta $\mathrm{T}$ cell receptor, the CD8 accessory molecule and preferentially uses the V delta 1 gene segment. Eur J Immunol. (1991) 21:1053-9. doi: 10.1002/eji.1830210429

99. Catalan-Serra I, Sandvik AK, Bruland T, Andreu-Ballester JC. Gammadelta $\mathrm{T}$ cells in crohn's disease: a new player in the disease pathogenesis? Journal of Crohn's and Colitis. (2017) 11:1135-45. doi: 10.1093/ecco-jcc/jjx039

100. Kühl AA, Loddenkemper C, Westermann J, Hoffmann JC. Role of gamma delta T cells in inflammatory bowel disease. Pathobiology. (2002) 70:150-5. doi: $10.1159 / 000068147$

101. Cosmi L, De Palma R, Santarlasci V, Maggi L, Capone M, Frosali F, et al. Human interleukin 17-producing cells originate from a CD161+CD4+ T cell precursor. J Exp Med. (2008) 205:1903-16. doi: 10.1084/jem.20080397

102. Kaser A, Ludwiczek O, Holzmann S, Moschen AR, Weiss G, Enrich B, et al. Increased expression of CCL20 in human inflammatory bowel disease. J Clin Immunol. (2004) 24:74-85. doi: 10.1023/B:JOCI.0000018066.46279.6b

103. Varona R, Cadenas V, Flores J, Martínez AC, Márquez G. CCR6 has a nonredundant role in the development of inflammatory bowel disease. Eur J Immunol. (2003) 33:2937-46. doi: 10.1002/eji.200324347

104. Fujino S, Andoh A, Bamba S, Ogawa A, Hata K, Araki Y, et al. Increased expression of interleukin 17 in inflammatory bowel disease. Gut. (2003) 52:65. doi: 10.1136/gut.52.1.65

105. Seiderer J, Elben I, Diegelmann J, Glas J, Stallhofer J, Tillack C. et al. Role of the novel Th17 cytokine IL-17F in inflammatory bowel disease (IBD): upregulated colonic IL-17F expression in active Crohn's disease and analysis of the IL17F pHis161Arg polymorphism in IBD. Inflamm Bowel Dis. (2008) 14:437-45. doi: 10.1002/ibd.20339

106. Kobayashi T, Okamoto S, Hisamatsu T, Kamada N, Chinen H, Saito R, et al. IL23 differentially regulates the Th1/Th17 balance in ulcerative colitis and Crohn's disease. Gut. (2008) 57:1682-9. doi: 10.1136/gut.2007.135053

107. Moschen AR, Tilg H. Raine IL-12 T, IL-23 and IL-17 in IBD: immunobiology and therapeutic targeting. Nat Rev Gastroenterol Hepatol. (2019) 16:185-96. doi: 10.1038/s41575-018-0084-8

108. Ogawa A, Andoh A, Araki Y, Bamba T, Fujiyama Y. Neutralization of interleukin-17 aggravates dextran sulfate sodium-induced colitis in mice. Clin Immunol. (2004) 110:55-62. doi: 10.1016/j.clim.2003.09.013

109. Wang J, Bhatia A, Krugliak Cleveland N, Gupta N, Dalal S, Rubin DT, et al. Rapid onset of inflammatory bowel disease after receiving secukinumab infusion. ACG Case Rep J. (2018) 5:e56. doi: 10.14309/cri.2018.56
110. Fauny M, Moulin D, Amico F, Netter P, Petitpain N, Arnone D, Jouzeau J-Y, Loeuille D, Peyrin-Biroulet L. Paradoxical gastrointestinal effects of interleukin-17 blockers. Ann Rheum Dis. (2020) 79:1132. doi: 10.1136/annrheumdis-2020-217927

111. Martin TM, Rosenbaum JT. An update on the genetics of HLA B27associated acute anterior uveitis. Ocul Immunol Inflamm. (2011) 19:108-14. doi: 10.3109/09273948.2011.559302

112. Wakefield D, Montanaro A, McCluskey P. Acute anterior uveitis and HLA-B27. Surv Ophthalmol. (1991) 36:223-32. doi: 10.1016/0039-6257(91)90005-Z

113. Khan MA, Kushner I, Braun WE. Association of HLA-A2 with uveitis in HLA-B27 positive patients with ankylosing spondylitis. J Rheumatol. (1981) 8:295-8.

114. Monowarul Islam SM, Numaga J, Fujino Y, Masuda K, Ohda H, Hirata R, et al. HLA-DR8 and acute anterior uveitis in ankylosing spondylitis. Arthritis Rheum. (1995) 38:547-50. doi: 10.1002/art.1780380414

115. Orchard TR, Chua CN, Ahmad T, Cheng H, Welsh KI, Jewell DP. Uveitis and erythema nodosum in inflammatory bowel disease: clinical features and the role of HLA genes. Gastroenterology. (2002) 123:714-8. doi: 10.1053 /gast.2002.35396

116. Loukil H, Kamoun A, Mahfoudh N, Frikha F, Snoussi M, Gaddour L, et al. Association study of MICA-TM and HLA-class I polymorphism with uveitis in South Tunisian population. Pathol Biol (Paris). (2015) 63:101-5. doi: 10.1016/j.patbio.2014.10.007

117. Goto K, Ota M, Maksymowych WP, Mizuki N, Yabuki K, Katsuyama Y, et al. Association between MICA gene A4 allele and acute anterior uveitis in white patients with and without HLA-B27. Am J Ophthalmol. (1998) 126:436-41. doi: 10.1016/S0002-9394(98)00100-7

118. Lindner E, Steinwender G, Plainer S, Poeschl EM, Weger M, Ardjomand $\mathrm{N}$, et al. Role of IL-10 gene polymorphisms in intermediate and HLA-B27-associated uveitis. Acta Ophthalmol. (2013) 91:e415-7. doi: 10.1111/aos.12104

119. Xiang Q, Chen L, Fang J, Hou S, Wei L, Bai L, et al. receptor-associated factor 5 gene confers genetic predisposition to acute anterior uveitis and pediatric uveitis. Arthritis Res Ther. (2013) 15:R113. doi: 10.1186/ar4293

120. Levinson RD. Killer immunoglobulin-like receptor genes in uveitis. Ocul Immunol Inflamm. (2011) 19:192-201. doi: 10.3109/09273948.2010.538798

121. Robinson PC, Claushuis TA, Cortes A, Martin TM, Evans DM, Leo P, et al. Genetic dissection of acute anterior uveitis reveals similarities and differences in associations observed with ankylosing spondylitis. Arthritis Rheumatol. (2015) 67:140-51. doi: 10.1002/art.38873

122. Zhou R, Caspi RR. Ocular immune privilege. F1000 Biol Rep. (2010) 2:3. doi: 10.3410/B2-3

123. Forrester JV, Kuffova L. Dick AAutoimmunity D, Autoinflammation, and Infection in Uveitis. Am J Ophthalmol. (2018) 189:77-85. doi: 10.1016/j.ajo.2018.02.019

124. Nakamura YK, Janowitz C, Metea C, Asquith M, Karstens L, Rosenbaum JT, et al. Short chain fatty acids ameliorate immune-mediated uveitis partially by altering migration of lymphocytes from the intestine. Sci Rep. (2017) 7:11745. doi: 10.1038/s41598-017-12163-3

125. Salvador RS, Horai R, Tang J, Zárate-Bladés C, Jittayasothorn Y, Itoh K, et al. Gut microbiota as a source of signals that trigger spontaneous ocular autoimmunity. J Immunol. (2017). 198:218.

126. McGonagle D, Aydin SZ, Gül A, Mahr A, Direskeneli H. 'MHC-Iopathy'-unified concept for spondyloarthritis and Behçet disease. Nat Rev Rheumatol. (2015) 11:731-40. doi: 10.1038/nrrheum.2015. 147

127. Reinhardt A, Yevsa T, Worbs T, Lienenklaus S, Sandrock I, Oberdörfer $\mathrm{L}$, et al. Interleukin-23-Dependent $\gamma / \delta \mathrm{T}$ Cells Produce Interleukin17 and Accumulate in the Enthesis. Aortic Valve, and Ciliary Body in Mice Arthritis. Rheumatol. (2016) 68:2476-86. doi: 10.1002/art. 39732

128. Jawad S, Liu B, Agron E, Nussenblatt RB, Sen HN. Elevated serum levels of interleukin-17A in uveitis patients. Ocul Immunol Inflamm. (2013) 21:434-9. doi: 10.3109/09273948.2013.815786

129. Amadi-Obi A, Yu CR, Liu X, Mahdi RM, Clarke GL, Nussenblatt RB, et al. TH17 cells contribute to uveitis and scleritis and are expanded by IL-2 and inhibited by IL-27/STAT1. Nat Med. (2007) 13:711-8. doi: 10.1038/nm1585 
130. Liu X, Lee YS Yu CR, Egwuagu CE. Loss of STAT3 in CD4+ T cells prevents development of experimental autoimmune diseases. J Immunol. (2008) 180:6070-6. doi: 10.4049/jimmunol.180.9.6070

131. Hohki S, Ohguro N, Haruta H, Nakai K, Terabe F, Serada S, et al. Blockade of interleukin-6 signaling suppresses experimental autoimmune uveoretinitis by the inhibition of inflammatory Th17 responses. Exp Eye Res. (2010) 91:162-70. doi: 10.1016/j.exer.2010.04.009

132. Caspi R. Autoimmunity in the immune privileged eye: pathogenic and regulatory $\mathrm{T}$ cells. Immunol Res. (2008) 42:41-50. doi: $10.1007 / \mathrm{s} 12026-008-8031-3$

133. Fotiadou C, Lazaridou E. Psoriasis and uveitis: links and risks. Psoriasis (Auckl). (2019) 9:91-6. doi: 10.2147/PTT.S179182

134. Gossec L, Baraliakos X, Kerschbaumer A, de Wit M, McInnes I, Dougados $\mathrm{M}$, et al. EULAR recommendations for the management of psoriatic arthritis with pharmacological therapies: 2019 update. Ann Rheum Dis. (2020) 79:700-12. doi: 10.1136/annrheumdis-2020-218456

135. Ritchlin CT, Kavanaugh A, Gladman DD, Mease PJ, Helliwell P, Boehncke WH, et al. Treatment recommendations for psoriatic arthritis. Ann Rheum Dis. (2009) 68:1387-94. doi: 10.1136/ard.2008.094946

136. Coates LC, Kavanaugh A, Mease PJ, Soriano ER, Laura Acosta-Felquer M, Armstrong AW, et al. Group for research and assessment of psoriasis and psoriatic arthritis (2015). Treatment recommendations for psoriatic arthritis. Arthritis Rheumatol. (2016) 68:1060-71. doi: 10.1002/art.39573

137. Reinink AR, Lee TC, Higgins PD. Endoscopic mucosal healing predicts favorable clinical outcomes in inflammatory bowel disease: a meta-analysis. Inflamm Bowel Dis. (2016) 22:1859-69. doi: 10.1097/MIB.0000000000000816

138. Turner D, Ricciuto A, Lewis A, D'Amico F, Dhaliwal J, Griffiths AM. et al. STRIDE-II: an update on the selecting therapeutic targets in inflammatory bowel disease (STRIDE) initiative of the international organization for the study of IBD (IOIBD): determining therapeutic goals for treat-to-target strategies in IBD. Gastroenterology. (2021) 160:1570-83. doi: 10.1053 /j.gastro.2020.12.031

139. Peyrin-Biroulet L, Sandborn W, Sands BE, Reinisch W, Bemelman W, Bryant RV, et al. Selecting therapeutic targets in inflammatory bowel disease (STRIDE): determining therapeutic goals for treat-to-target. Am J Gastroenterol. (2015) 110:1324-38. doi: 10.1038/ajg.2015.233

140. Ungaro R, Colombel JF, Lissoos T, Peyrin-Biroulet L, A. Treat-to-target update in ulcerative colitis: a systematic review. Am J Gastroenterol. (2019) 114:874-83. doi: 10.14309/ajg.0000000000000183

141. Colombel JF, D'Haens G, Lee WJ, Petersson J, Panaccione R. Outcomes and strategies to support a treat-to-target approach in inflammatory bowel disease: a systematic review. J Crohns Colitis. (2020) 14:254-66. doi: 10.1093/ecco-jcc/jjz131

142. Khoudari G, Mansoor E, Click B, Alkhayyat M, Saleh MA, Sinh P, et al. Rates of intestinal resection and colectomy in inflammatory bowel disease patients after initiation of biologics: a cohort study. Clin Gastroenterol Hepatol. (2020). doi: 10.1016/j.cgh.2020.10.008

143. Gomollón F, Dignass A, Annese V, Tilg H, Van Assche G, Lindsay JO, et al. ECCO, 3rd European evidence-based consensus on the diagnosis and management of crohn's disease 2016: part 1: diagnosis and medical management. J Crohn's Colitis. (2016) 11:3-25. doi: 10.1093/ecco-jcc/jjw168

144. Lichtenstein GR, Loftus EV, Isaacs KL, Regueiro MD, Gerson LB, Sands $\mathrm{BE}$, et al. Clinical guideline: management of crohn's disease in adults. Am J Gastroenterol. (2018) 113:481-517. doi: 10.1038/ajg.2018.27

145. Torres J, Bonovas S, Doherty G, Kucharzik T, Gisbert JP, Raine T, et al. ECCO guidelines on therapeutics in crohn's disease: medical treatment. J Crohns Colitis. (2020) 14:4-22. doi: 10.1093/ecco-jcc/jjz180

146. Akobeng AK, Zachos M. Tumor necrosis factor-alpha antibody for induction of remission in Crohn's disease. Cochrane Database System Rev. (2003) 4. doi: 10.1002/14651858.CD003574.pub2

147. Behm BW. Bickston SJ. Tumor necrosis factor-alpha antibody for maintenance of remission in Crohn's disease. Cochrane Database System Rev. (2008) 1. doi: 10.1002/14651858.CD006893

148. Hanauer SB, Sandborn WJ, Rutgeerts P, Fedorak RN, Lukas M, MacIntosh D, et al. Human anti-tumor necrosis factor monoclonal antibody (adalimumab) in Crohn's disease: the CLASSIC-I trial. Gastroenterology. (2006). 130:32333. doi: 10.1053 /j.gastro.2005.11.030
149. Sandborn WJ, Hanauer SB, Rutgeerts P, Fedorak RN, Lukas M, MacIntosh DG, et al. Adalimumab for maintenance treatment of Crohn's disease: results of the CLASSIC II trial. Gut. (2007) 56:1232-9. doi: 10.1136/gut.2006.106781

150. Sandborn WJ, Rutgeerts P, Enns R, Hanauer SB, Colombel JF, Panaccione R, et al. Adalimumab induction therapy for Crohn disease previously treated with infliximab: a randomized trial. Ann Intern Med. (2007) 146:829-38. doi: 10.7326/0003-4819-146-12-200706190-00159

151. Colombel JF, Sandborn WJ, Rutgeerts P, Enns R, Hanauer SB, Panaccione $\mathrm{R}$, et al. Adalimumab for maintenance of clinical response and remission in patients with Crohn's disease: the CHARM trial. Gastroenterology. (2007) 132:52-65. doi: 10.1053/j.gastro.2006.11.041

152. Rutgeerts P, Van Assche G, Sandborn WJ, Wolf DC, Geboes K, Colombel $\mathrm{JF}$, et al. Adalimumab induces and maintains mucosal healing in patients with Crohn's disease: data from the EXTEND trial. Gastroenterology. (2012). 142:1102-11.e2. doi: 10.1053/j.gastro.2012.01.035

153. Targan SR, Hanauer SB, van Deventer SJ, Mayer L, Present DH, Braakman $\mathrm{T}$, et al. A short-term study of chimeric monoclonal antibody cA2 to tumor necrosis factor alpha for Crohn's disease. Crohn's disease cA2 study group. $N$ Engl J Med. (1997) 337:1029-35. doi: 10.1056/NEJM199710093371502

154. Hanauer SB, Feagan BG, Lichtenstein GR, Mayer LF, Schreiber S, Colombel JF, et al. Maintenance infliximab for Crohn's disease: the ACCENT I randomised trial. Lancet. (2002) 359:1541-9. doi: 10.1016/S0140-6736(02)08512-4

155. Sands BE, Anderson FH, Bernstein CN, Chey WY, Feagan BG, Fedorak RN, et al. Infliximab maintenance therapy for fistulizing Crohn's disease. $N$ Engl J Med. (2004) 350:876-85. doi: 10.1056/NEJMoa030815

156. Colombel JF, Sandborn WJ, Reinisch W, Mantzaris GJ, Kornbluth A, Rachmilewitz D, et al. Infliximab, azathioprine, or combination therapy for Crohn's disease. N Engl J Med. (2010) 362:1383-95. doi: 10.1056/NEJMoa0904492

157. Sandborn WJ, Feagan BG, Stoinov S, Honiball PJ, Rutgeerts P, Mason D, et al. Certolizumab pegol for the treatment of Crohn's disease. N Engl J Med. (2007) 357:228-38. doi: 10.1056/NEJMoa067594

158. Schreiber S, Colombel JF, Bloomfield R, Nikolaus S, Schölmerich J, Panés $\mathrm{J}$, et al. Increased response and remission rates in short-duration Crohn's disease with subcutaneous certolizumab pegol: an analysis of PRECiSE 2 randomized maintenance trial data. Am J Gastroenterol. (2010) 105:1574-82. doi: $10.1038 /$ ajg. 2010.78

159. Sandborn WJ, Abreu MT, D'Haens G, Colombel JF, Vermeire S, Mitchev K, et al. Certolizumab pegol in patients with moderate to severe Crohn's disease and secondary failure to infliximab. Clin Gastroenterol Hepatol. (2010). 8:688-95.e2. doi: 10.1016/j.cgh.2010.04.021

160. Targan SR, Feagan B, Vermeire S, Panaccione R, Melmed GY, Landers C, et al. A Randomized, double-blind, placebo-controlled phase 2 study of brodalumab in patients with moderate-to-severe Crohn's disease. Am J Gastroenterol. (2016) 111:1599-607. doi: 10.1038/ajg.2016.298

161. Sandborn WJ, Gasink C, Gao LL, Blank MA, Johanns J, Guzzo C, et al. Ustekinumab induction and maintenance therapy in refractory Crohn's disease. $N$ Engl J Med. (2012) 367:1519-28. doi: 10.1056/NEJMoa120 3572

162. Feagan BG, Sandborn WJ, Gasink C, Jacobstein D, Lang Y, Friedman JR, et al. Ustekinumab as Induction and Maintenance Therapy for Crohn's Disease. New Engl J Med. (2016) 375:1946-60.

163. Feagan BG, Sandborn WJ, D'Haens G, Panés J, Kaser A, Ferrante M, et al. Induction therapy with the selective interleukin-23 inhibitor risankizumab in patients with moderate-to-severe Crohn's disease: a randomised, doubleblind, placebo-controlled phase 2 study. Lancet. (2017) 389:1699-709. doi: 10.1016/S0140-6736(17)30570-6

164. Sandborn WJ, Ghosh S, Panes J, Vranic I, Wang W, Niezychowski W. A phase 2 study of tofacitinib, an oral Janus kinase inhibitor, in patients with Crohn's disease. Clin Gastroenterol Hepatol. (2014). 12:1485-93.e2. doi: 10.1016/j.cgh.2014.01.029

165. Panés J, Sandborn WJ, Schreiber S, Sands BE, Vermeire S, D’Haens G, et al. Tofacitinib for induction and maintenance therapy of Crohn's disease: results of two phase IIb randomised placebo-controlled trials. Gut. (2017) 66:1049-59. doi: 10.1136/gutjnl-2016-312735

166. Vermeire S, Schreiber S, Petryka R, Kuehbacher T, Hebuterne X, Roblin $\mathrm{X}$, et al. Clinical remission in patients with moderate-to-severe Crohn's 
disease treated with filgotinib (the FITZROY study): results from a phase 2, double-blind, randomised, placebo-controlled trial. Lancet. (2017) 389:26675. doi: 10.1016/S0140-6736(16)32537-5

167. Sandborn WJ, Feagan BG, Loftus EV, Peyrin-Biroulet L, Van Assche G, D'Haens G, et al. Efficacy and safety of upadacitinib in a randomized trial of patients with Crohn's disease. Gastroenterology. (2020). 158:2123-38.e8. doi: 10.1053/j.gastro.2020.01.047

168. Lichtenstein GR, Yan S, Bala M, Hanauer S. Remission in patients with Crohn's disease is associated with improvement in employment and quality of life and a decrease in hospitalizations and surgeries. Am J Gastroenterol. (2004) 99:91-6. doi: 10.1046/j.1572-0241.2003.04010.x

169. Feagan BG, Panaccione R, Sandborn WJ, D’Haens GR, Schreiber S, Rutgeerts PJ, et al. Effects of adalimumab therapy on incidence of hospitalization and surgery in Crohn's disease: results from the CHARM study. Gastroenterology. (2008) 135:1493-9. doi: 10.1053/j.gastro.2008.07.069

170. Echarri A, Ollero V. Barreiro-de Acosta M, Fernández-Villaverde A, Hernández V, Lorenzo A, et al. Clinical, biological, and endoscopic responses to adalimumab in antitumor necrosis factor-naive Crohn's disease: predictors of efficacy in clinical practice. Eur J Gastroenterol Hepatol. (2015) 27:430-5. doi: 10.1097/MEG.0000000000000296

171. Mastronardi M, Curlo M, Cavalcanti E, Burattini O, Cuppone R, Tauro $\mathrm{R}$, et al. Administration timing is the best clinical outcome predictor for adalimumab administration in Crohn's disease. Frontiers in medicine. (2019) 6:234-234. doi: 10.3389/fmed.2019.00234

172. Sandborn WJ, Feagan BG, Rutgeerts P, Hanauer S, Colombel JF, Sands $\mathrm{BE}$, et al. Vedolizumab as induction and maintenance therapy for Crohn's disease. N Engl J Med. (2013) 369:711-21. doi: 10.1056/NEJMoa121 5739

173. Sands BE, Feagan BG, Rutgeerts P, Colombel JF, Sandborn WJ, Sy R, et al. Effects of vedolizumab induction therapy for patients with Crohn's disease in whom tumor necrosis factor antagonist treatment failed. Gastroenterology. (2014). 147:618-27.e3. doi: 10.1053/j.gastro.2014.05.008

174. AbbVie. Risankizumab (SKYRIZI@) Demonstrates Significant Improvements in Clinical Remission and Endoscopic Response in Two Phase 3 Induction Studies in Patients with Crohn's Disease. (2021). Available online at: https://news.abbvie.com/news/press-releases/risankizumabskyrizi-demonstrates- significant-improvements-in-clinical-remissionand-endoscopic-response-in-two-phase-3-induction-studies-in-patientswith-crohns-disease.htm. (accessed July 1, 2021)

175. AbbVie. Phase 3 Maintenance Results Show Patients with Crohn's Disease Receiving Risankizumab (SKYRIZI $®$ ) Achieved Endoscopic Response and Clinical Remission at One Year. (2021). Available online at: https:// news.abbvie.com/news/press-releases/phase-3-maintenance-results-showpatients-with-crohns-disease-receiving-risankizumab-skyrizi-achievedendoscopic-response-and-clinical-remission-at-one-year.htm. (accessed July 1, 2021)

176. Hueber W, Sands BE, Lewitzky S, Vandemeulebroecke M, Reinisch W, Higgins PD, et al. Secukinumab, a human anti-IL-17A monoclonal antibody, for moderate to severe Crohn's disease: unexpected results of a randomised, double-blind placebo-controlled trial. Gut. (2012) 61:1693-700. doi: 10.1136/gutjnl-2011-301668

177. Genovese M C, Colombel J, Gellett A M., Xu W, D H. Incidence of inflammatory bowel disease among patients treated with ixekizumab: an update on adjudicated data from an integrated database of patients with psoriasis and psoriatic arthritis. In ACR/ARHP Annual Meeting. 2018: Arthritis Rheumatol. (2018).

178. Reinisch W, Sandborn WJ, Hommes DW, D’Haens G, Hanauer S, Schreiber $\mathrm{S}$, et al. Adalimumab for induction of clinical remission in moderately to severely active ulcerative colitis: results of a randomised controlled trial. Gut. (2011) 60:780-7. doi: 10.1136/gut.2010.221127

179. Sandborn WJ, van Assche G, Reinisch W, Colombel JF, D'Haens G, Wolf DC, et al. Adalimumab induces and maintains clinical remission in patients with moderate-to-severe ulcerative colitis. Gastroenterology. (2012). 142:25765.e1-3. doi: 10.1053/j.gastro.2011.10.032

180. Rutgeerts P, Sandborn WJ, Feagan BG, Reinisch W, Olson A, Johanns J, et al. Infliximab for induction and maintenance therapy for ulcerative colitis. $N$ Engl J Med. (2005) 353:2462-76. doi: 10.1056/NEJMoa 050516
181. Panaccione R, Ghosh S, Middleton S, Márquez JR, Scott BB, Flint L, et al. Combination therapy with infliximab and azathioprine is superior to monotherapy with either agent in ulcerative colitis. Gastroenterology. (2014). 146:392-400.e3. doi: 10.1053/j.gastro.2013.10.052

182. Sandborn WJ, Feagan BG, Marano C, Zhang H, Strauss R, Johanns J, et al. Subcutaneous golimumab induces clinical response and remission in patients with moderate-to-severe ulcerative colitis. Gastroenterology. (2014). 146:85-95. doi: 10.1053/j.gastro.2013.05.048

183. Sandborn WJ, Feagan BG, Marano C, Zhang H, Strauss R, Johanns J, et al. Subcutaneous golimumab maintains clinical response in patients with moderate-to-severe ulcerative colitis. Gastroenterology. (2014). 146:96109.e1. doi: 10.1053/j.gastro.2013.06.010

184. Feagan BG, Rutgeerts P, Sands BE, Hanauer S, Colombel J-F, Sandborn WJ, et al. Vedolizumab as induction and maintenance therapy for ulcerative colitis. New Engl J Med. (2013) 369:699-710. doi: 10.1056/NEJMoa121 5734

185. Sands BE, Peyrin-Biroulet L, Loftus EV, Danese S, Colombel J-F, Törüner $\mathrm{M}$, et al. Vedolizumab versus adalimumab for moderate-to-severe ulcerative colitis. New Engl J Med. (2019) 381:1215-26. doi: 10.1056/NEJMoa190 5725

186. Sandborn WJ, Su C, Sands BE, D'Haens GR, Vermeire S, Schreiber S, et al. Tofacitinib as Induction and Maintenance Therapy for Ulcerative Colitis. New Engl J Med. (2017) 376:1723-36. doi: 10.1056/NEJMoa160 6910

187. AbbVie. A Study to Evaluate the Long-Term Safety and Efficacy of Upadacitinib (ABT-494) in Participants With Ulcerative Colitis (UC). (2021). Available online at: https:// clinicaltrials.gov/ct2/show/NCT03006068. (accessed July 1, 2021)

188. Babu K, Mahendradas P. Medical management of uveitis - current trends. Indian J Ophthalmol. (2013) 61:277-83. doi: 10.4103/0301-4738.114099

189. Cordero-Coma M, Sobrin L. Anti-tumor necrosis factor- $\alpha$ therapy in uveitis. Surv Ophthalmol. (2015). 60:575-89. doi: 10.1016/j.survophthal.2015.06.004

190. Jaffe GJ, Dick AD, Brézin AP, Nguyen QD, Thorne JE, Kestelyn P, et al Adalimumab in patients with active noninfectious uveitis. $N$ Engl J Med. (2016) 375:932-43. doi: 10.1056/NEJMoa1509852

191. Nguyen QD, Merrill PT, Jaffe GJ, Dick AD, Kurup SK, Sheppard J, et al. Adalimumab for prevention of uveitic flare in patients with inactive noninfectious uveitis controlled by corticosteroids (VISUAL II): a multicentre, double-masked, randomised, placebo-controlled phase 3 trial. Lancet. (2016) 388:1183-92. doi: 10.1016/S0140-6736(16)31339-3

192. van Bentum RE, Heslinga SC, Nurmohamed MT, Gerards AH, Griep EN, Koehorst C, et al. Reduced occurrence rate of acute anterior uveitis in ankylosing spondylitis treated with golimumab - The GO-EASY study. J Rheumatol. (2019) 46:153-9. doi: 10.3899/jrheum.180312

193. Van der Horst-Bruinsma I, Van Bentum R, Verbraak F, Rath $T$, Rosenbaum J, Misterska-Skora M, et al. THU0379 reduction of anterior uveitis flares in patients with axial spondyloarthritis following one year of treatment with certolizumab pegol: 48-week interim results from a 96-week open-label study. Ann Rheum Dis. (2020) 79:423-423. doi: 10.1136/annrheumdis-2020-eular.3747

194. Dick AD, Tugal-Tutkun I, Foster S, Zierhut M, Melissa Liew SH, Bezlyak V, et al. Secukinumab in the treatment of noninfectious uveitis: results of three randomized, controlled clinical trials. Ophthalmology. (2013) 120:777-87. doi: 10.1016/j.ophtha.2012.09.040

195. Letko E, Yeh S, Foster CS, Pleyer U, Brigell M, Grosskreutz CL. Efficacy and safety of intravenous secukinumab in noninfectious uveitis requiring steroidsparing immunosuppressive therapy. Ophthalmology. (2015) 122:939-48. doi: 10.1016/j.ophtha.2014.12.033

196. Sen HN. Ustekinumab (STELARA) for the Treatment of Active SightThreatening Uveitis (STAR Study). Available online at: https://clinicaltrials. gov/ct2/show/results/NCT02911116. (accessed July 5, 2021)

197. Terrier, Efficacy B. Safety of Ustekinumab, a Human Monoclonal Anti-IL12/IL-23 Antibody, in Patients With Behçet Disease (STELABEC). Available online at: https://clinicaltrials.gov/ct2/show/NCT02648581. (accessed July 5, 2021)

198. Braun J, Baraliakos X, Listing J, Sieper J. Decreased incidence of anterior uveitis in patients with ankylosing spondylitis treated with the anti-tumor 
necrosis factor agents infliximab and etanercept. Arthritis Rheum. (2005) 52:2447-51. doi: 10.1002/art.21197

199. Visalli E, Crispino N, Foti R. Multidisciplinary management of psoriatic arthritis: the benefits of a comprehensive approach. Adv Ther. (2019) 36:806-16. doi: 10.1007/s12325-019-00 901-0

200. Cañete JD, Puig L. Multidisciplinary teams for psoriatic arthritis: on aims and approaches. Actas Dermosifiliogr. (2014) 105:325-7. doi: 10.1016/j.adengl.2014.01.002

Conflict of Interest: The authors declare that the research was conducted in the absence of any commercial or financial relationships that could be construed as a potential conflict of interest.
Publisher's Note: All claims expressed in this article are solely those of the authors and do not necessarily represent those of their affiliated organizations, or those of the publisher, the editors and the reviewers. Any product that may be evaluated in this article, or claim that may be made by its manufacturer, is not guaranteed or endorsed by the publisher.

Copyright (c) 2021 Chia, Ang, Chan, Chan, Chong and Leung. This is an open-access article distributed under the terms of the Creative Commons Attribution License (CC $B Y)$. The use, distribution or reproduction in other forums is permitted, provided the original author(s) and the copyright owner(s) are credited and that the original publication in this journal is cited, in accordance with accepted academic practice. No use, distribution or reproduction is permitted which does not comply with these terms. 